ACCEPTED MANUSCRIPT

\title{
Discharge dynamics, plasma kinetics and gas flow effect in argon- acetylene discharges
}

To cite this article before publication: Gautier Tetard et al 2021 Plasma Sources Sci. Technol. in press https://doi.org/10.1088/1361-6595/ac2a17

\section{Manuscript version: Accepted Manuscript}

Accepted Manuscript is "the version of the article accepted for publication including all changes made as a result of the peer review process, and which may also include the addition to the article by IOP Publishing of a header, an article ID, a cover sheet and/or an 'Accepted

Manuscript' watermark, but excluding any other editing, typesetting or other changes made by IOP Publishing and/or its licensors"

This Accepted Manuscript is @ 2021 IOP Publishing Ltd.

During the embargo period (the 12 month period from the publication of the Version of Record of this article), the Accepted Manuscript is fully protected by copyright and cannot be reused or reposted elsewhere.

As the Version of Record of this article is going to be / has been published on a subscription basis, this Accepted Manuscript is available for reuse under a CC BY-NC-ND 3.0 licence after the 12 month embargo period.

After the embargo period, everyone is permitted to use copy and redistribute this article for non-commercial purposes only, provided that they adhere to all the terms of the licence https://creativecommons.org/licences/by-nc-nd/3.0

Although reasonable endeavours have been taken to obtain all necessary permissions from third parties to include their copyrighted content within this article, their full citation and copyright line may not be present in this Accepted Manuscript version. Before using any content from this article, please refer to the Version of Record on IOPscience once published for full citation and copyright details, as permissions will likely be required. All third party content is fully copyright protected, unless specifically stated otherwise in the figure caption in the Version of Record.

View the article online for updates and enhancements. 


\title{
Abstract
}

\author{
G. Tetard ${ }^{1}$, A. Michau ${ }^{1}$, S. Prasanna ${ }^{1}$, J. Mougenot ${ }^{1}$, P. Brault $^{2}$, K. Hassouni ${ }^{1}$ \\ ${ }^{1}$ Université Sorbonne Paris Nord, Laboratoire des Sciences des Procédés et des Matériaux, LSPM, CNRS, UPR \\ 3407, F-93430, Villetaneuse, France
${ }^{2}$ GREMI CNRS -Université d'Orléans, BP744 Orléans Cedex 2, France \\ 3407, F-93430, Villetaneuse, France
${ }^{2}$ GREMI CNRS -Université d'Orléans, BP744 Orléans Cedex 2, France
}

\section{Discharge dynamics, plasma kinetics and gas flow effect in argon-acetylene discharges}

\section{Abstract}

We investigated capacitively coupled $\mathrm{Ar} / \mathrm{C}_{2} \mathrm{H}_{2} \mathrm{RF}$ plasmas with a 1D fluid model that couples a 13.56 $\mathrm{MHz}$ discharge module, a long timescale chemical module and a flow transport module. A new solution procedure was developed in order to accurately describe the coupling between the short timescale discharge dynamics and the long characteristic time processes that play a major role in the molecular growth of reactive species. The plasma was simulated for different inlet gas configurations and flowrates. We showed that for a showerhead configuration one may distinguish two situations. For short residence time the plasma was strongly electronegative in the very center of the discharge gap and dominated by large hydrocarbon positive and negative ions. In this situation the acetylene conversion, although moderate, lead to a significant molecular growth. For long residence time, although $\mathrm{C}_{2} \mathrm{H}_{2}$ underwent a total conversion, the products of the primary $\mathrm{C}_{2} \mathrm{H}_{2}$ dissociation process were consumed by surface deposition which reduced drastically the molecular growth in the short gap discharge considered here. Whatever the conditions, we confirmed the key-role of $\mathrm{Ar}^{*}$ in the acetylene conversion, ionization kinetics as well as the subsequent molecular growth for neutral and charged species. We also showed that remote feed gas and showerhead configurations predicted similar results at low flowrate. At larger flowrate the two configurations presented some discrepancy. Especially $\mathrm{H}_{2}$ density was much larger for the remote feed gas configuration, which affected the overall plasma behavior. Our results highlight that realistic gas-flow models are essential for an accurate description of acetylene conversion in $\mathrm{Ar} / \mathrm{C}_{2} \mathrm{H}_{2}$ plasma.

\section{Introduction}

Capacitively coupled RF (CCRF) discharges in hydrocarbon (HC) containing mixtures have been the subject of interest for many decades [1], [2], [3]. These discharges were indeed used fairly early for the deposition of a variety of (multi-)functional carbon, i.e., Diamond-Like-carbon(DLC) [4] or TaC [5], and hydrogen-carbon coatings $/(\mathrm{aC}: \mathrm{H})$ [6] with applications in a large number of fields such as tribology [7], diffusion barriers [8], anti-corrosion coatings [9], adhesion-enhancement [6], nanocomposites elaboration [10], biomedical [11], just to cite few examples. Although the carbon deposits elaborated by HC-containing discharges enable reaching a large number of functionalities, the physical properties and functional characteristics of the deposits are usually highly sensitive to the process characteristics. Consequently, a fairly tight tuning of the deposition process is very often necessary in order to achieve targeted functional properties [12], [13]. This tuning requires investigating the effect of a significant number of process parameters, such as the nature of the $\mathrm{HC}$ 
precursors, the feed gas composition, the pressure, the reactor configuration, the flow rate, the RF voltage magnitude, the gap distance etc. This makes a process optimization based on a simple parametric analysis hardly achievable in many situations, especially when taking into account the fairly rich (and sometime complex) physical phenomena and chemical processes that govern HCcontaining discharge plasmas. This complexity motivated extensive experimental and modeling studies. Special attention was devoted to plasma-surface interaction, molecular growth, particle nucleation, dust particle dynamics and their impact on film deposition and/or nanostructures formation [14]. Beside coatings applications, the interest in these discharges was also motivated by the fundamental understanding of the production of analogs to extraterrestrial carbonaceous compounds and particles [15] on one hand and the issues of dust formation in tokamak fusion edgeplasma [16]. In particular, molecular growth and nanoparticle formation in acetýlene and argon/acetylene RF discharges is receiving a constant attention, since more than two decades [1], [17], [18]. Concentrating on argon-acetylene CCRF plasmas, extensive experimental studies have been carried out to (i) investigate the space-time distributions of dust formation in the continuous or the pulsed regimes by laser light scattering and absorption [19],[20], (ii) thoroughly analyze the space-time evolution of both particle size and density by combination of Kinetic Mie ellipsometry and laser extinction [21], (iii) monitor hydrocarbon concentrations and analyze the chemical kinetics and the discharge dynamics by mass spectrometry and Tunable diode Laser Absorption spectroscopy [22],[23],[24],[25], (iv) investigate the initial clustering processes that induce particle nucleation by mass and molecular beam spectrometry [26] [27], (v) analyze the anion dynamics in the early stage of the discharge [28], (vi) study the particle cloud dynamics and its coupling with the discharge characteristics [29], [28], [30] and (vii) shed light on the role of argon metastables that turns out to be a key-species as far discharge dynamics and plasma kinetics are concerned [31], [32]. Besides these studies that were specifically dedicated to heterogeneous gas phase kinetics and plasma dynamics in $\mathrm{Ar}-\mathrm{C}_{2} \mathrm{H}_{2}$ CCRF discharges, an experimental effort was also devoted to the investigation of the interaction of the plasma species and the growing deposit. These experiments provided the probabilities for sticking, recombination and, to a much lesser extent, more complex surfacereactions mechanism on a:C-H for some $\mathrm{HC}$ species [16], [33] , which helps in describing the plasmasurface interaction and the coupling between surface processes, the discharge dynamics and the plasma composition. The large amount of key-information brought by these experiments enabled gaining insight in some of the processes that govern argon-acetylene CCRF discharges, giving guidelines for model development and validation. Actually, model developments were first carried out on pure acetylene CCRF plasmas with a first contribution from Doyle [34] and Stoykov et al. [35] who proposed a kinetic model taking into account the formation of large $\mathrm{HCs}, \mathrm{C}_{n>2} \mathrm{H}_{\mathrm{y}}$, in low pressure acetylene plasmas. These first models were used in the frame of quasi-homogeneous plasma assumption to investigate the clustering processes that leads to nanosized particle formation. They showed that although nucleation likely takes place through linear HC growth, the contribution of large Polycyclic Aromatic Hydrocarbon may be significant. Many steps forward have been achieved by Bogaerts and coworkers who integrated detailed chemical kinetic in one-dimensional plasma simulations where the discharge dynamics is properly taken into account [36]. This enables them analyzing under more realistic space-time distribution of the charged species and electrontemperature several molecular growth routes that may contribute to particle nucleation [37-40]. Beside these models that targeted the analysis of the combined molecular growth and discharge dynamics phenomena, other authors focused on the investigation of some aspects of the aerosol dynamics [37] such as coagulation, [10; 41]. This effort on acetylene plasma was followed few years 
later by the development of numerical models for argon-acetylene CCRF plasmas. The first models were proposed by Schweigert and coworkers who investigated the impact of the growing nanoparticles on the discharge characteristics [42] , the change in an argon discharge behavior when acetylene is added as impurity by the integration of a detailed chemical model inspired from those developed for acetylene in one-dimensional discharge model [43]. Much more recently, Denysenko and coworkers made use of a quasi-homogeneous model to investigate the chemical kinetics in RF argon-acetylene plasmas [44; 45]. In particular these authors emphasized the role of argon metastables on the discharge kinetics and analyzed the transient evolution of the plasma.

A close look to the literature reveals that argon-acetylene CCRF plasmas show many peculiarities with a large number of questions that have still to be answered as far as modeling is concerned.

As a matter of fact, experimental studies addressing different discharge aspects, particle formation, argon-metastables evolution, composition change, negative ion dynamic, etc. clearly showed that argon-acetylene plasma may show very long time-evolution, i.e. up to several tens of seconds for some effects. As a result, these plasmas are characterized by a very stiff dynamic with characteristic times that range between sub-nanoseconds, for the electron energy dynamics, and several seconds for large species or particles. This is for example the case of the addition of ethynil radical $\left(\mathrm{C}_{2} \mathrm{H} \bullet\right)$ on polyynes [39]. Similarly the mutual neutralization between positive and negative ions shows characteristic times of the order of $10^{-2} \mathrm{~s}$ The complexity brought by this stiffness is further increased by the necessity to resolve the RF period, i.e., $10^{-7} \mathrm{~s}$, while describing the tight coupling that may exist between phenomena or processes with very different characteristic times. To our best knowledge, this issue has never been explicitly addressed in the literature. In particular, the way the very fast discharge dynamics is coupled to the much slower molecular growth kinetics or large negative ions transport has never been thoroughly discussed. This point is especially critical when considering the strong coupling between the fairly slow molecular growth and attachment kinetics that end up with the formation of large negative ions and the very fast discharge dynamic that is affected by these ions. Therefore, in this paper, we discuss a new solution procedure that enables taking into account such coupling. Beside the classical balance equations used in fluid models, this procedure makes use of a set of non-stationary short time-scale averaged equations where almost all the species can be followed over long time scales.

Further, the long time-evolution, from several to tens of seconds, of acetylene and/or argon/acetylene plasmas raises the question on the effect of the feed gas flow velocity and configuration on the plasma behavior. In particular, one may expect that some collisional effects or transport phenomena may show characteristic times that are of the same order of magnitude as the residence time. In such a case the discharge behavior cannot be affected by the only flowrate value but also by the flow configuration. As a matter of fact, CCRF argon-acetylene plasmas were generated under both showerhead electrode and remote feed gas configurations with flowrate ranging between 1 and $20 \mathrm{scm}$ and residence estimated between $500 \mathrm{~ms}$ and $50 \mathrm{~s}$ depending on the discharge gap and the reactor volume [46], [47], [48]. Obviously, such differences in the residence time and the flow configuration may result in different plasma compositions and discharge characteristics even for the same working pressure feed gas composition and RF voltage. We believe therefore that the flow effect on the plasmas composition and discharge behavior should be thoroughly investigated. In particular, many experiments showed a very large conversion of acetylene, i.e., more than 90\%, in argon-acetylene plasmas [32], [24],[25]. Such a high conversion yield raises the question on the fate of acetylene and the nature of hydrocarbon species that actually interact with electrons. Although previous modeling works showed a significant conversion of 
acetylene and gave indication on the resulting species, we believe that this question needs further analysis. In particular, we will show that the conversion yield of acetylene and the discharge behavior strongly change with the flowrate and the feed gas inlet configuration. Lastly, from more fundamental point of view, the extensive work of Stefanovic and coworkers on argon-acetylene CCRF plasmas emphasizes the key role of argon metastables in argon-acetylene discharges [31],[32]. This effect has been taken into account in one-dimensional model in [43] and was also very recently underlined and thoroughly analyzed through modeling by Denysenko et al. [45] who used a volume averaged plasma model. We propose in this article to analyze argon metastable effect while taking into account more accurate discharge dynamics. We propose in particular to investigate how this species is involved in the interplay between discharge dynamic, plasma kinetics, gas flow convective transport.

In this article, we mainly focus on the investigation of flow effect during the molecular growth stage prior to any solid particle formation which is therefore not considered in the present model.

We make use of a one-dimensional self-consistent numerical fluid model of argon-acetylene plasmas along with a new solution procedure where a set of short-time-scale averaged transport equations are considered in order to track the long-time-scale evolution of the plasma. The model selfconsistently solves for the discharge dynamic equations (fluid model), flow momentum equations for showerhead inlet stagnation flow configuration and short-time-scale averaged transport equations for chemical species. We also propose and discuss a set of non-linear mass-conserving boundary conditions that are consistent with stagnation flow configuration and enable properly taking into account mass transfer during $\mathrm{HC}$ deposition. A special effort was indeed devoted to insure the mass conservation without introducing any normalization or correction procedure so as to insure an accurate $\mathrm{Ar} / \mathrm{C} / \mathrm{H}$ balance in these highly reactive discharges. The developed model was used to investigate how the flow velocity and configuration affect the plasma behavior in terms of composition, key-species and governing processes. We particularly show that the neutral plasma composition dramatically change with the flow velocity and may strongly differ from the feed gas composition. We underline how the ionization kinetics, the discharge electronegativity and the acetylene conversion yield change with the flow velocity. We compare three different flow configurations: a first one where the feed gas composition is assumed at the showerhead electrode, a second one where the back-diffusion and surface reaction at the showerhead electrode are taken into account and a third one where a remote feed gas configuration is assumed.

In the next section the fluid model used is presented with special emphasis on (i) the short-time-scale averaged transport equations used to track chemical species over long time-scales, (ii) the flow models used to describe the showerhead and the remote feed gas inlet configurations and (iii) the boundary conditions used. The discharge drift-diffusion and chemistry models used are essentially taken from the literature and are therefore described more briefly with a special emphasis on the change/improvement that we made in this work. In section 3 we present the main results obtained on the effect of the gas flow on the discharge behavior. We first analyze in detail the plasmas obtained when using a convective flow boundary conditions at the showerhead electrode, i.e. when the composition at the showerhead electrode is assumed to be the same as the feed gas composition. Then, we discuss the case where more realistic boundary conditions taking into account the back-diffusion and surface reaction at the showerhead electrode are taken into account. In this section we will also show how the flow velocity affects the plasma behavior. Then, we compare the plasmas compositions obtained for showerhead and remote flow inlet configurations. A brief 
These two sub-models have to be complemented by a fluid-flow model that gives the flow velocity and/or the gas flow related convection fluxes in the gap. These fluxes may indeed bring a substantial contribution in the species and energy balance equations, especially for large characteristic time species. The three sub-modules and the dedicated solution procedure used in the present work are presented in the following.

We are especially interested in describing how the gas flow may affect the plasma composition and subsequently the discharge dynamic and the detailed chemistry that leads to nucleation. Although the dust particle formation is not taken into account, the chemical model involves a large number of species and reactions and shows strong stiffness. Therefore, in order to keep the numerical solution tractable, we consider a one-dimensional model where the plasma composition is investigated on the central axis along the gap between the powered and the grounded electrodes. Such a onedimensional model is especially suitable when the gap distance is small as compared to the electrode diameter.

\section{2. Discharge dynamic model}

summary of the results and the major conclusions and perspectives that may be drawn from this work are given in the last section.

\section{Model description}

The acetylene plasmas generated in the capacitive coupled RF discharges show very large stiffness since the characteristic time of the discharge dynamic is below $10^{-7} \mathrm{~s}$ while the chemistry may take place over tens of seconds when molecular growth and particle formation occur [19]. In order to treat such stiffness, one may separate the species in two groups according to their characteristic times. The first group considers the species with chemistry/transport characteristic times that are below or of the order of the RF period. The second group gathers those species with characteristic times much greater than the RF period. This time-scale separation would naturally results in a plasma period time-scale and a second sub-module called Large characteristic time transport/chemistry module (LCT Module)_that describes the coupled phenomena of chemistry and transport taking place

One of the major issues encountered when using this separation is related to the coupling between the two time-scales and more specifically to the feedback of large time-scale evolution of the plasma composition on the short time-scale discharge dynamics. This issue is particularly difficult to address in the case of long time-scale variations of the charged species, especially negative ions. Therefore, small time-scale dynamics of these ion species within a RF period and the LCT module considers a time averaged representation of these ions to predict their evolution over longer time-scales. The coupling between the two sub-modules is enforced/by the iterative exchange of these species until convergence. 
The discharge module solves for the charged species densities, the electron energy and the electric field over a time scale shorter than the $13.56 \mathrm{MHz}$ RF period. For this purpose we used a classical fluid model assuming a drift/diffusion transport [49] that involves a mass conservation equation, expressed in terms of mass fraction $Y_{s,}$ for each plasma species :

$$
\rho \frac{d Y_{S}}{d t}=-\frac{d \Gamma_{S}}{d x}+S_{s}+F_{S}
$$

Where $\rho$ is the gas density, while $Y_{s}, \Gamma_{\mathrm{S}}$ and $S_{s}$ represent the mass fraction, the drift/diffusion flux and the net mass production rate by collisional processes for species ' $s$ '. The term $F_{S}$ represents the contribution of the gas flow convection flux in the balance of species ' $s$ '. It may involve either a flux divergence term or a source term depending on the flow configuration and model used. This term will be discussed in section II.3.

The low inertia electrons follow the RF field dynamic. The drift-diffusion flux is given by the local time-varying electric field $E$. We therefore have:

$$
\Gamma_{e}=-D_{e} \rho \frac{Y_{e}}{x_{e}} \frac{d x_{e}}{d x}-\mu_{e} \mathrm{E} \rho Y_{e}
$$

Where $D_{e}$ and $\mu_{e}$ are the diffusion and mobility coefficients for electrons.

The ionic species have much larger inertia and their oscillations under the action of the RF field may be strongly dumped. In principle, a momentum equation has to be considered for each ion in order to determine the pressure/electrostatic forces generated ion transport velocity and flux. This would however increase the size of the governing equations set, which would introduce major difficulties in their numerical solution. We therefore used the simplified procedure proposed in [49], where a driftdiffusion transport model using an effective electric field $E_{s}$ derived from the damping equations is adopted for each ion ' $s$ ' :

$$
\frac{d E_{S}}{d t}=-v_{Q M-S}\left(E_{S}-E\right)
$$

Where $V_{Q M-s}$ the momentum exchange collision frequency for the ion ' $s$ '. The ion flux is then expressed using drift-diffusion equation similar to (2) where the effective electric field is used instead of the electric field.

The time varying local electric field is determined taking into account the plasma space charge using Poisson's equation.

The expressions of the net mass production rates by collisional processes for the different species depend on the chemical model and rate constant data used. The reaction rate constants and the transport coefficients involved in the species balance equations highly depend on the gas temperature and the electron energy distribution function (eedf). In the present model the gas temperature $T_{g}$ is assumed to be constant and is treated as a model input parameter. On the other hand, the eedf may be well approximated by the solution of the two-term expansion of the Boltzmann equation since the investigated plasmas are fairly collisional. We then make use of an offline Boltzmann solver to determine the eedf, the resulting electron temperature, $T_{e}=\frac{2}{3} \frac{\left\langle\varepsilon_{e}\right\rangle}{k_{b}}$, and transport coefficients over a wide range of reduced electric field value. The electron-impact reaction rate constants and electron transport coefficients were curve-fitted as function of the electron temperature. These curve-fits were used in the plasma model in order to self-consistently determine 


\section{3. Large characteristic time transport/chemistry module (LCT Module)}

As mentioned previously, many of the neutral species involved in the hydrocarbon plasma chemistry show characteristic times (in the order of $1 \mathrm{~s}$ ) much longer that the RF period $\left(\sim 10^{-7} \mathrm{~s}\right)$. These species may affect the discharge dynamic over a very long time scale. Describing this effect by integrating the transport equations of these species within the discharge module is computationally expensive. Therefore, we have developed a second module that is specific to long characteristic time species and where the considered equations are derived from the time-average of the species continuity equations over one RF period. These may be expressed as follows:

$$
<\rho \frac{d Y_{S}}{d t}>_{T_{R F}}=-\frac{d<\vec{\Gamma}_{S}>_{T_{R F}}}{d x}+<S_{S}>_{T_{R F}}+<F_{S}>_{T_{R F}}
$$

Where $\left\langle f>_{T_{R F}}(t)=\frac{1}{T_{R F}} \int_{t-\frac{T_{R F}}{2}}^{t+\frac{T_{R F}}{2}} f(t) d t\right.$ is the time-average of $f$ over one RF period. Here we are interested in the time-variation of $\langle f\rangle_{T_{R F}}$ over time-scales much longer than the RF period.

Actually, most of the neutral species (the only exception is $\mathrm{Ar}^{*}$ ) show transport and chemistry characteristic-times that are much longer than the RF period so as $\left\langle Y_{S}\right\rangle_{T_{R F}}(t) \approx Y_{S}$. Similarly the change in the gas density remains moderate and takes place on time scales that are much longer than the RF period so as $\left\langle\rho>_{T_{R F}}(t) \approx \rho\right.$. Also, the transport fluxes for these species only include constant or slowly varying quantities. This means that for the neutral species:

and

$$
\left\langle\Gamma_{S}\right\rangle_{T_{R F}}=\left\langle-D_{S} \rho \frac{Y_{S}}{x_{s}} \frac{d x_{s}}{d x}\right\rangle_{T_{R F}} \approx-D_{s} \rho \frac{Y_{s}}{x_{s}} \frac{d x_{s}}{d x}
$$

$$
<F_{S}>_{T_{R F}} \approx F_{S}
$$

As for the source term $S_{s}$, one may distinguish two components. A first one, $S_{s-e}$, that involves electron-impact processes, and a second one, $S_{s-h s}$, that involves only heavy species. The rates of reactions between heavy species depend on their densities that are generally slowly varying and on the constant gas temperature that determines the rate constants. This means that we have $\left\langle S_{s-h s}\right\rangle=S_{s-}$ hs. The electron-impact component of the source term may be written as: 


$$
S_{s-e}=\sum_{r} \alpha_{r} k_{r}\left(T_{e}\right) n_{e} n_{p}
$$

Where ' $p$ ' is the species that react with the electron in reaction ' $r$ ', $k_{r}\left(T_{e}\right)$ is the rate constant of process ' $r$ ', $\alpha_{r}$ is a constant coefficient that depends on the contribution of species ' $s$ ' in reaction ' $r$ '. For most cases, the only factors that vary very rapidly in the expression of $S_{s-e}$ are the rate constant that depends on $T_{e}$ and the electron density. The expression of the time-averaged value of $S_{s-e}$ over one RF period may be written:

$$
<S_{s-e}>_{T_{R F}}=\sum_{r} \alpha_{r}<k_{r}\left(T_{e}\right) n_{e}>_{T_{R F}} n_{p}
$$

Taking into account the discussion above, the expression of time-averaged continuity equations of long characteristic-time (LCT) species may be written as:

$$
\rho \frac{d Y_{S}}{d t}=-\frac{d \Gamma_{s}}{d x}+S_{s-h s}+<S_{s-e}>_{T_{R F}}+F_{S}
$$

The electron-impact component of the source term is evaluated in the discharge dynamic module discussed in the previous section.

Actually, the procedure discussed above for neutral species may be also used in the case of ionic species that show very small time-space variation during a single RF cycle. This is especially the case for the low mobility large ions that are produced during the molecular growth. It is also the case for the negative ions, the evolution of which is at least partly governed by the fairly long ion-ion mutual neutralization process. For this purpose we solved long time evolution of these species by introducing image-ion in this sub-module. The time-averaging procedure of the continuity equations for these image-ions is similar to that for neutrals except that the drift terms needs to be averaged also. If constant diffusion and mobility coefficients are assumed for the ions, the averaged driftdiffusion flux may be expressed as:

$$
\left\langle\Gamma_{s}\right\rangle_{R F}=-D_{s} \rho \frac{Y_{s}}{x_{s}} \frac{d x_{s}}{d x}-\mu_{e}\left\langle E_{s}\right\rangle_{R F} \rho Y_{e}
$$

The time-averaged effective electric field $\left\langle E_{S}\right\rangle_{R F}$ is equal to the time-averaged electric field and corresponds therefore to the DC component of the field, i.e., $\left\langle E_{S}\right\rangle_{R F}=\langle E\rangle_{R F}=-\frac{d V_{D C}}{d x}$.

The $\left\langle E_{s}\right\rangle_{R F}$ required in the $L C T$ Module is therefore obtained from the discharge module.

\section{4. Gas-flow models}

The flow field in CCRF plasma reactors is generally fairly complex and its detailed description would require 3D flow modeling. However, if one is only interested in the flow field inside the gap region, two model-configurations that correspond to either showerhead feed gas inlet or remote can be treated in the frame of a 1D model. In the first configuration, the flow enters the simulation domain axially through one of the electrode. The flow shows a stagnation line configuration that requires solving for the axial velocity profile in the discharge gap. In the second situation where a remote feed gas inlet is used, the feed gas is introduced across the lateral surface of the gap over the entire interelectrode domain. 


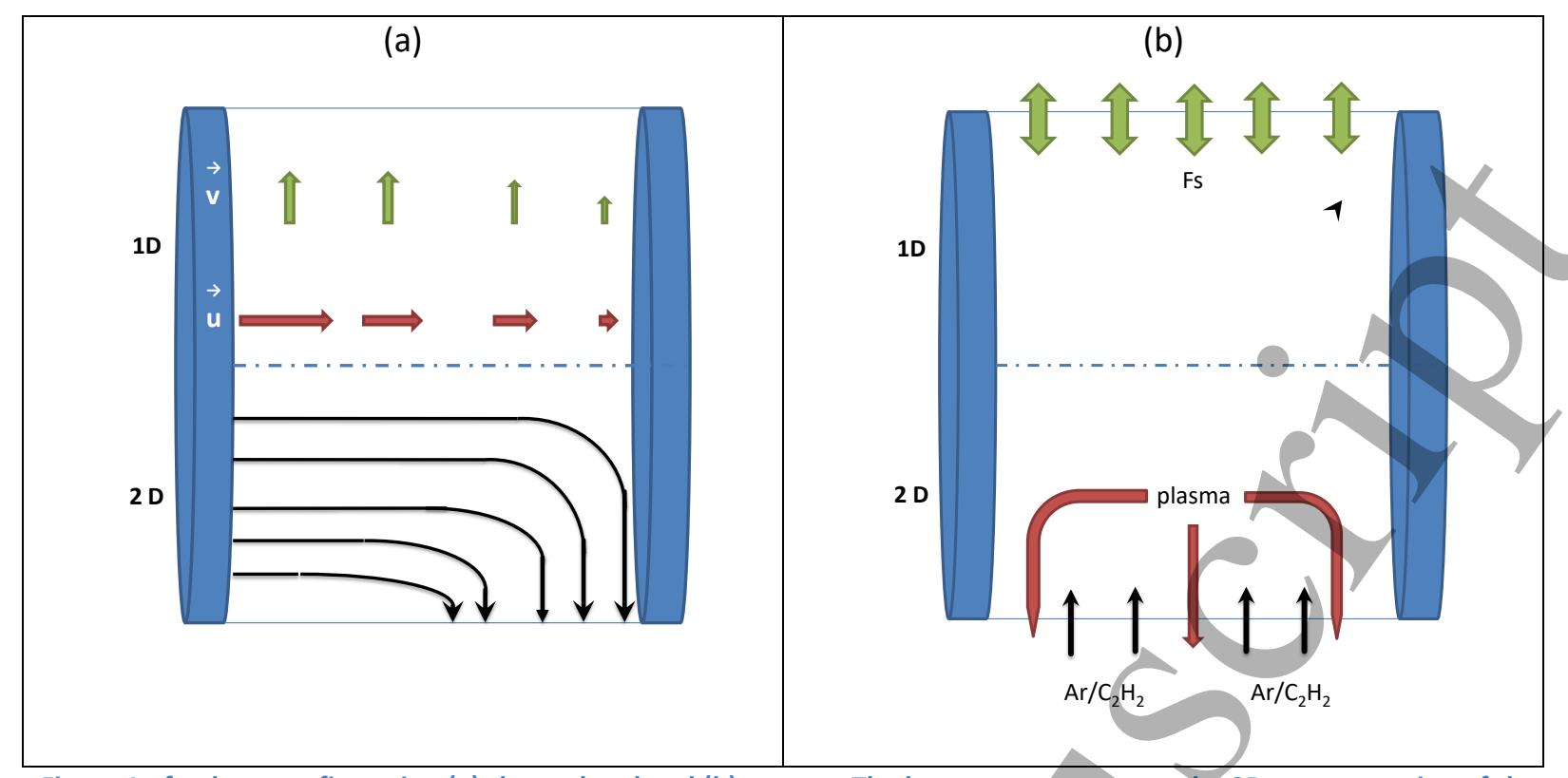

Figure 1 : feed gas configuration (a) showerhead and (b) remote. The lower part represents the 2D representation of the flow and the upper part the 1D equivalent. $\vec{u}$ and $\vec{v}$ are the axial and radial velocity components of the flow for the showerhead feed gas configuration and $F_{s}$ is the flow contribution to the mass balance equation (equation 15 for the showerhead feed gas configuration and equation 16 for the remote gas inlet configuration).

\section{- Showerhead gas inlet configuration}

The first situation corresponds to a showerhead inlet and a stagnation flow configuration. In this case we approximate the showerhead electrode (left electrode in figure 1) by a porous body from which a purely axial flow emerges and is directed toward the facing electrode (the Stagnation Electrode) which then locally stops the flow. In this stagnation point configuration, the flow exits radially outside the inter-electrode domain. Coltrin et al [50] proposed a simple mathematical model for the description of such stagnation flow configuration. This model assumes a parabolic stream function and a constant radial component of the pressure gradient. The resulting variation of the axial velocity along the stagnation line, i.e., the gap, is described by two physical quantities, the axial velocity $u$, and the so-called acceleration factor at the stagnation line: $v=\frac{v}{r}$ where $v$ is the radial velocity. The flow velocity and acceleration factor in the gap are governed by the following set of continuity and radial momentum conservation equations [51]:

$$
\begin{gathered}
\frac{d \rho u}{d x}+2 \rho V=0 \\
\rho u \frac{\partial V}{\partial x}+\rho V^{2}=-\Lambda+\frac{d}{d x}\left(\eta \frac{d V}{d x}\right)
\end{gathered}
$$

Where $\eta$ is the dynamic viscosity and $\Lambda=\frac{1}{r} \frac{\partial P}{\partial r}$ is a constant that characterizes the stagnation flow. In the considered finite-gap situation, $\Lambda$ is determined iteratively to satisfy the imposed velocity values $u_{e l e c}$ at the boundary. The contribution of the flow rate, $F_{s}$, to the species mass balance equations (1) and (11) includes two components: a convective flux divergence term that describes the convective transport along the gap and a source term corresponding to the radial convective losses along the stagnation line. This contribution may be written: 


$$
F_{s}=-\frac{d \rho u Y_{s}}{d x}+Y_{s} \frac{d \rho u}{d x}
$$

\section{- Remote feed gas inlet configuration}

The way the effect of convective flow is treated in the literature corresponds approximately to this configuration [43]. Actually, in this configuration, the flow effect will strongly depend on the ratio of the discharge to the reactor volumes, $\delta=\frac{V_{D}}{V_{R}}$, that determines the residence time in the discharge gap. A literature survey shows that this parameter is generally not precisely indicated. Estimates from the data given on experimental CCRF plasma reactors in some references would indicate that this parameter may vary by almost one order of magnitude from one case to another [44],[47]. Therefore, in this work we considered two asymptotic cases. The first one corresponds to $\delta \rightarrow 0$, which means that the discharge volume is very small with respect to the reactor volume. In this case the composition outside the discharge volume would not be affected by the discharge and would be similar to the feed gas composition. The situation corresponds therefore to an infinite dilution of the discharge products in the surrounding reactor volume. The gas flow effect may be described through a simple uniform mass transfer along the lateral surface of the discharge. The flow contribution to the species balance equations may be expressed:

$$
F_{s}=h_{s}\left(\rho_{s}-\rho_{s}^{\text {feed }}\right) \frac{4 /{ }^{\prime}}{D_{\text {electrode }}}
$$

Where $h_{s}$ is a mass transfer coefficient, $h_{s}=\frac{D_{S}}{D_{\text {electrode }}}, D_{\text {electrode }}$ is the electrode diameter, and $\rho_{s}^{\text {feed }}$ is the species density in the feed gas.

The second situation corresponds to $\delta \rightarrow 1$, which means that the discharge occupies the entire reactor. In this case, the inlet mass flow of species ' $s$ ' corresponds to the mass flow of ' $s$ ' in the feed gas. The outlet mass flow for species ' $s$ ' is given by the product of its mass fraction in the discharge by the total mass flow exiting the reactor. The flow contribution to the species balance equations is written:

$$
F_{s}=\frac{\left(Q_{m}^{\text {inlet }} Y_{s}^{\text {feed }}-Q_{m}^{\text {out }} Y_{s}\right)}{V_{\text {plasma }}}
$$

$Y_{S}^{f e e d}$ denotes the mass fraction of the different species in the feed gas. $V_{\text {plasma }}$ is the discharge volume. $Q_{m}^{\text {inlet }}$ and $Q_{m}^{\text {out }}$ are the inlet and outlet mass flowrates. They may differ since deposition on the electrodes may take place.

\section{5. Chemistry model, collisional and transport data}

We have used a chemistry model that is essentially similar to the ones discussed in references [39] and [44]. We will give only a brief description of the main processes considered. The electron impact reaction-set involves (i) ionization of $A r, A r *[52], \mathrm{C}_{2} \mathrm{H}_{2}$ and $\mathrm{H}_{2}$, (ii) dissociation of $\mathrm{C}_{2} \mathrm{H}_{2}$ and $\mathrm{H}_{2}$ and (iii) dissociative attachment processes of $\mathrm{C}_{2} \mathrm{H}_{2}$. Acetylene ionization and dissociation also takes place through quenching by $\mathrm{Ar}^{*}$ which, due to its key-role [32], is considered as a chemical species in the model, The rate constant used from these processes are taken from [53] and correspond to 
ionization/dissociation branching ratio of 0.7/0.3. More recently, Stefanovic et al suggested the inverse value, i.e., $0.3 / 0.7$, that was determined from the comparison between measured and calculated time decays of the electron density during the afterglow of an RF discharge [32] Therefore the sensitivity of our results to this parameter was investigated. The first series of primary reactions discussed above results in the formation of (i) small ions, i.e., $\mathrm{Ar}^{+}, \mathrm{C}_{2} \mathrm{H}_{2}{ }^{+}, \mathrm{C}_{2} \mathrm{H}^{+}, \mathrm{C}_{2} \mathrm{H}^{-}$,(ii) radicals, i.e., $\mathrm{C}_{2} \mathrm{H}, \mathrm{C}_{2}, \mathrm{CH}$, and (iii) a significant amount of molecular and atomic hydrogen. Both negative and positive primary hydrocarbon ions produced by this set of reactions undergo a series of ionconversion reactions by collisions with $\mathrm{C}_{2} \mathrm{H}_{2}$ and $\mathrm{C}_{2} \mathrm{H}$, which results in molecular growth and formation of large positive, $\mathrm{C}_{n} \mathrm{H}_{y}{ }^{+}$, and negative, $\mathrm{C}_{n} \mathrm{H}^{-}$ions with even number of carbon [48]. In the present model we describe this molecular growth up to 8 carbons. The considered neutral kinetics is very similar to the one described in reference [39]. It particularly includes neutral molecular growth route through addition of $\mathrm{C}_{2} \mathrm{H}_{2}$ and $\mathrm{C}_{2} \mathrm{H}$ which results in the formation of large $\mathrm{C}_{n} \mathrm{H}_{2}$ polyyne molecules and the corresponding $\mathrm{C}_{n} \mathrm{H}$ radicals. The large negative-ions and neutral molecules are coupled through dissociative attachment of polyynes [54] [55] and radiative attachment on $\mathrm{C}_{n} \mathrm{H}$ radicals [56]. They are also coupled through positive-negative ion mutual neutralization processes which represent the only loss process for the negative ions. The positive ion and neutral species kinetics are coupled through Penning ionization of acetylene by $\mathrm{Ar}^{*}[32]$, dissociative recombination of positive ions, positive-negative ion mutual neutralization processes and surface recombination.

Beside these reactive processes we have also considered a set of collisional processes that are involved in the electron energy dissipation and do not affect the plasma composition. In particular, we took into account (i) the excitations of the first three vibrational levels and the first two nondissociative electronically excited states of $\mathrm{H}_{2}$ that are responsible for the major part of electron energy dissipation through e- $\mathrm{H}_{2}$ collisions, (ii) the excitation of the three vibrational levels and the electronically excited states of $\mathrm{C}_{2} \mathrm{H}_{2}$ [43] and (iii) the excitation of argon radiative states [52].

The mobility coefficients for ions were determined using Langevin theory and Blanc's mixing law [57] assuming that the background gas is essentially composed of argon, $\mathrm{C}_{2} \mathrm{H}_{2}$ and hydrogen. The ion diffusion coefficients $D_{j}$ (in $\mathrm{m}^{2} . \mathrm{s}^{-1}$ ) of the ionic species are inferred from the mobility coefficient using Einstein equation [58]. The diffusion coefficients of the neutral species were estimated using Lennard-Jones parameters and a Blanc's mixing law where the background gas is assumed to be dominated by argon, acetylene and hydrogen.

\section{6. Boundary conditions}

In order to solve the species continuity, the electron energy and Poisson's equations, boundary conditions are to be specified at the electrodes.

As for Poisson's equation the voltage values at the powered and the grounded electrodes are given, i.e., $V(x=0)=V_{0} \cdot \sin (\omega t)$ and $V(x=L)=0$

The species boundary conditions at the electrodes used in this work takes into account the possibility of a gas flow through the electrodes in order to describe the showerhead configuration on one hand and surface reactions on the other hand. They express the balance between the net species flux toward the electrode and the net mass of species consumption by surface reactions at the electrode. This may be written: 


$$
\begin{aligned}
& \left(-D_{s} \rho \frac{Y_{s}}{x_{s}} \frac{\mathrm{dx}_{s}}{\mathrm{dx}}\right)_{\text {elec }}+\left(\mathrm{u}_{d-s}+u_{\text {elec }}\right)\left(\rho Y_{s}\right)_{\text {elec }}-\left(\rho_{\text {feed }} y_{s, f e e d} \overrightarrow{u_{\text {feed }}}\right)_{\text {elec }} \\
& =\left.(\gamma+\sigma) v_{s}\right|_{\text {elec }}-\left.w_{s}^{+}\right|_{\text {elec }}
\end{aligned}
$$

The LHS of equation (18) gathers the flux terms involved in the boundary conditions. The first term of the LHS is the diffusion flux at the electrode. The second term represents the advection flux due to both gas flow $\left(u_{\text {elec }}\right)$ and drift transport $\left(u_{d-s}\right)$ at the electrode. The third term represents the mass flux of species ' $s$ ' that flows in from the electrode with the feed gas inlet. This term is non zero only at a showerhead electrode, i.e., $\left.u_{\text {feed }}\right|_{\text {elec }} \neq 0$, and for argon and acetylene species.

The RHS of equation (18) represents the net loss of species ' $s$ ' by surface chemistry. The first term represents the loss rate by recombination processes and sticking (deposition) to the electrode surface. $\left.v_{s}\right|_{\text {elec }}$ is the collision frequency of species ' $s$ ' per unit area with the electrode, while $\gamma$ and $\sigma$ are the recombination and sticking probabilities. We consider that stable species (i.e. Argon, molecular hydrogen and polyynes) are reflected at the wall. Positive ions totally recombine at the wall through dissociative processes $(\gamma=1)$ while negative ones are trapped into the bulk and do not interact with the surface. With regard to the radicals, it is well known that the recombination coefficient greatly depends on the processing conditions such as temperature or surface composition [33], [59], [16]. Such variations could greatly impact the deposition process but have limited influence on the discharge dynamics as shown in [60]. Therefore, we have adopted constant values of sticking coefficients. Following the recommendation of [16] small recombination coefficient of $10^{-}$ ${ }^{3}$ has been used for radicals while values of 1 from [49] have been used for Ar*.

The second term, $\left.w_{s}^{+}\right|_{\text {elec }}$ represents the mass production rate of ' $s$ ' by surface chemistry, i.e. from recombination of other species.

The collision frequency $\left.v_{s}\right|_{\text {elec }}$ depends on the nature of the species i.e. if it is charged or neutral. For neutral species $v_{s} l_{\text {elec }}$ is obtained from the thermal velocity. The ions however are strongly accelerated in the sheath which significantly enhanced the drift velocity and the collision frequency [61] , [62].

It is worthy to mention that the flow velocity at the electrode $u_{\text {elec }}$ in equation (18) is different from the inlet velocity of the feed gas $u_{\text {feed }}$. This is due to the fact that the net mass flow at the electrode includes not only feed gas mass flow but also a net mass flux that results from species back-diffusion and surface reactions. Actually, the contribution of the feed gas flow to the boundary conditions (18) appears as a linear source term and not as a flux term. The value of $u_{e l e c}$ is to be determined from a total mass balance equation at the electrode. Such an equation is obtained by summing equation (18) over all the chemical species. When performing such a summation, the terms corresponding to diffusion fluxes, drift fluxes, mass production rates and recombination rates cancel out. This leads to the following equation that determines the velocity at the electrode:

$$
u_{\text {elec }}=\frac{\rho_{\text {feed }}}{\rho} \cdot u_{\text {feed }}-\left.\frac{1}{\rho} \sum \sigma v_{s}\right|_{\text {elec }}
$$

In fact, equation (19) specifies the boundary conditions for the flow velocity at the electrodes and it appears once again that $u_{e l e c}$ is not necessarily equal to $u_{\text {feed }}$. The use of this equation is of critical importance in order to insure accurate total mass balance and relative predominance of the three elements $\mathrm{C} / \mathrm{H} / \mathrm{Ar}$.

All the species boundary conditions that should be in principle applied in order to determine the molar fraction of each species at the electrode, $Y_{S}$ lelec.can be derived from equations (18) and (19). The most simple and commonly used boundary condition in the literature [43], [37] , [39], may be obtained by neglecting diffusion and drift transport in LHS of equation (18) as well as surface 


\section{7. Solution procedure}

The solution of the equations that describe the discharge dynamics, the transport/chemistry of long characteristic time species and the flow velocity component is performed using an iterative procedure. At each iteration step, the discharge dynamic equations, i.e., the species continuity equations for electron and ions, the electron energy conservation equation, the Poisson's equation subject to their boundary conditions, are first time-integrated over several hundreds of RF periods in order to approach the permanent regime. During this first step the densities of neutral species are assumed to be constant. The time-averaged collision frequencies for electron-impact processes required in the LCT module are inferred from this first step. Then, the second step consists in the time-integration of the LCT species continuity equations coupled to the steady state flow velocity equations. Here the coupling between the species and flow equations is of prime importance since the surface reactions play a role in the velocity boundary conditions. The flow velocity equations are solved iteratively so as to adjust the pressure gradient in order to fulfill the boundary conditions on the velocity. This second step is dedicated to LCT species evolution. It will obviously concern the neutral species as well as some ions. As a matter of fact, in some conditions, i.e. high discharge electronegativity, ions may show long time-scale evolutions due to some non-linear slow processes such as mutual neutralization. Actually, it is very difficult to proceed to a complete separation between ions that are treated in the discharge dynamic module and neutral species treated in the LCT module. It is necessary to keep considering all the ions in the discharge dynamic model because even a small change or modulation of the ion density may strongly affect the space charge field and alter the resulting self-consistent field. This double-constraint led us to keep integrating the ion continuity equations in the discharge dynamic module and to introduce for each ion an image-ion that is treated over much longer time scale using the time-averaged continuity equations (Equation (6)). Each image-ion has exactly the same characteristics and undergoes the same chemistry as the corresponding ion. The only difference between an ion and its image is that, the continuity equation is integrated over the RF period time scale for an ion, while time-averaged continuity equation is used for the image-ion. The ion densities in the discharge module are corrected using the image ion density computed in the LCT module along with a convenient under-relaxation.

The iterative procedure described above is carried out until the permanent discharge regime and steady-state for LCT species densities are reached. In such conditions, the time-averaged density of 
an ion and the density of the corresponding image-ion should be the same. A special attention was devoted to check this equality which is one of the convergence criteria.

\section{Results and discussion}

\section{1. Considered discharges conditions}

One of the main objectives of this study is to analyze the effect of the flow velocity and configuration on the plasma characteristics. We focus on the following discharge conditions: a pressure of $p=10$ $\mathrm{Pa}$, an electrode diameter of $20 \mathrm{~cm}$, a gap distance of $2.54 \mathrm{~cm}$, an RF voltage amplitude, $\mathrm{V}_{\mathrm{RF}}=100 \mathrm{~V}$, and a molar feed gas composition of $4 \%$ acetylene and $96 \%$ argon. These conditions are close to those considered in reference [39] and [43] .

We will first investigate $\mathrm{Ar} / \mathrm{C}_{2} \mathrm{H}_{2}$ plasma behavior for a showerhead configuration with a flow rate of $10 \mathrm{sccm}$, which corresponds to a flow inlet velocity of $5 \times 10^{-2} \mathrm{~m} \cdot \mathrm{s}^{-1}$, assuming purely convective boundary conditions at the showerhead electrode. The details of the transport phenomena and collisional processes that govern energy depositions, ionization kinetics, discharge dynamics and plasma compositions will be thoroughly analyzed for this first base-case. Then, in the second part of the study we will use the more realistic mixed convection-diffusion-surface reaction boundary conditions. We will show how such a change in the boundary conditions affects not only the plasma composition but also the plasma dynamics. Then, we will investigate the effect of the flow velocity on the plasma characteristics under showerhead feed gas configuration. Eventually, comparison between the showerhead and remote feed gas configurations will be carried out.

\section{2. Convective boundary conditions at the showerhead electrode - acetylene dominated}

\section{discharges.}

In this first part we analyze the $\mathrm{Ar} / \mathrm{C}_{2} \mathrm{H}_{2}$ discharge when using a purely convective boundary condition at the showerhead electrode located at $\mathrm{x}=0 \mathrm{~m}$, with the mole fractions set to 0.96 for argon, $4 \times 10^{-2}$ for $\mathrm{C}_{2} \mathrm{H}_{2}$ and $\mathrm{O}$ for all other neutral species. The boundary conditions at the other electrode takes into account the surface processes and are given by equation (Equation 18) with $u_{\text {feed }}=0$.

The axial profiles of time-averaged electron density and temperature are given in Figure 2 . The electron density in the bulk of the plasma is as high as $3 \times 10^{15} \mathrm{~m}^{-3}$, a value that is closed to the one measured by Stefanovic et al. [32]. Similar electron density value are also predicted by Ariskin et al. [43] who used a 1D hybrid model and Denysenko et al. [45] who used quasi-homogeneous, i.e., OD, model. The average electron temperature reaches approximately $4 \mathrm{eV}$ in the plasma bulk and $5 \mathrm{eV}$ in the sheath. These values are in good agreement with those usually reported for Argon discharges under similar conditions [64] and those reported by Greiner et al. [65]. These values are however much higher than those reported by Ariskin et al. [43], i.e. $1 \mathrm{eV}$ in the bulk and $1.6 \mathrm{eV}$ in the sheath. The instantaneous values of electron temperature may be much larger. They reach a peak-value of $15 \mathrm{eV}$ in the sheath during the cathodic phase. The average electron temperature shows nonmonotonous behavior with a first increase inside the sheath and a decrease very close to the electrode. The temperature variation inside the sheath is well resolved in the simulation, while the steep decrease at the electrode could be hardly resolved even with highly refined mesh. Nevertheless, this has no impact on the simulation results since (i) the temperature variation remains 
moderate,(ii) there is very weak ionization and energy dissipation very near the electrode and (iii) the instantaneous electron temperature axial profiles are very well resolved in the sheaths except very close to the electrode during the cathodic phase when the electron density is very small in this region.

These differences may be explained by several factors. The gap size in [43] is much larger than that used in this work, which would result in reduced surface losses. The electron temperature required for sustaining the discharge would be therefore much lower. The second reason is also related to a significant difference in the electron loss rate. As a matter of fact, it seems that Ariskin et al. do not take into account the attachment on large $\mathrm{HC}$ which represents a significant electron loss channel in our model. A last reason for this difference is related to the treatment of the electron kinetics in the two approaches. Ariskin et al. make use of hybrid model and a kinetic approach that enables taking into account the stochastic heating effect that results in an enhanced production of high energy electrons. This is not the case in our model where we assume that the eedf satisfies the two-term expansion of the Boltzmann equation, which leads to a collisional depletion of the high energy electron population. As a result the predicted value for the electron temperature necessary for sustaining the discharge obtained from a hybrid model would be naturally smaller than that predicted by a fluid model. Note however that a major difference between the present model and Ariskin et al.'s is related to the central role of argon metastable in the ionization kinetics. It seems to us that electron impact ionization on $\mathrm{Ar}^{*}$ and ionization of $\mathrm{C}_{2 \mathrm{n}} \mathrm{H}_{2}$ through $\mathrm{Ar}^{*}$ quenching, that represent a major contribution in the overall ionization kinetics in our study, are not taken into account by Ariskin et al. Omitting Ar* mediated ionization processes should in principle lead to a larger electron temperature. This is obviously not the case here and it seems that even these processes are important, the difference between the electron temperature values predicted by the two models is determined by the assumption made on the eedf and/or the gap distance effect and/or the attachment kinetics. The electron temperature predicted by the present fluid model is also larger than that of Denysenko et al. [45] who used a OD model with Druyvesteyn eedf. This is in principle similar to the eedf determined in this work by solving the Boltzmann equation. The difference in the electron temperature is therefore more probably due to the difference in the electron loss term that is spatially averaged in Denysenko et al. global model. In any case this discussion shows that comparison between the electron temperature values obtained by the models mentioned above is difficult and many factors may explain the differences observed.

Erreur ! Source du renvoi introuvable. 2 shows the power density, in W. $\mathrm{m}^{-3}$, gained by the electrons from the electric field, $W_{R F}$, and the power density that the electrons dissipate through collisions, $Q_{c o l}$. We observe that the RF power is deposited over a fairly large region extending from the sheath to the bulk of the plasma. The maximum power deposition is however obtained in the regions around the sheath edges, the deposited RF power density is fairly small near the electrodes and in the center of the plasma. We notice that the collisional dissipation power density value, $Q_{c o l}$ is consistent with the value reported in [43]. It is dominated by the non-dissociative $\mathrm{C}_{2} \mathrm{H}_{2}$ excitation. The total power deposited in the whole discharge gap is approximately $6 \mathrm{~W}$ for an electrode diameter of $20 \mathrm{~cm}$. It appears that the power density dissipated by the electrons in collisional processes is much lower than the power they gain from the RF field. Also, the axial profile of the dissipated power density is very different from the RF power deposition profile. It shows a maximum in the center of the discharge and very weak values in the vicinity of the electrodes. This emphasizes the non-local character of electron energy relaxation and the important role of electron energy 
transport as far as the discharge equilibrium is concerned. From Figure 2 we could estimate that only $45 \%$ of the power is dissipated in the plasma chemistry through the collision term $Q_{c o l}$. The remaining power is lost by the electron absorbed at the electrodes.

A detailed analysis of the collision term $Q_{c o l}$ provides the main routes of collisional power dissipation used to sustain the plasma. It turns out that more than $50 \%$ of the collisional power dissipation takes place through the excitation of the argon metastables state. More than $20 \%$ is due to the electronic excitation of $\mathrm{C}_{2} \mathrm{H}_{2}$ and only $\approx 20 \%$ of this power is used for the electron impact ionization of $\mathrm{C}_{2} \mathrm{H}_{2}$ and Ar. Actually, the argon metastables plays a key-role in the discharge equilibrium. Figure 4 shows the axial profiles of $\mathrm{Ar} *$ molar fraction calculated in the investigated $\mathrm{Ar} / \mathrm{C}_{2} \mathrm{H}_{2}$ discharge and in a pristine argon discharge for the same $\mathrm{RF}$ voltage, pressure and gap distance. It appears that the metastables density in $\mathrm{Ar} / \mathrm{C}_{2} \mathrm{H}_{2}$ discharge is two orders of magnitude lower than in pristine Argon discharge, i.e. $8 \times 10^{15} \mathrm{~m}^{-3}$ and $8 \times 10^{17} \mathrm{~m}^{-3}$ respectively. This is due to the very fast quenching of $\mathrm{Ar}^{*}$ by $\mathrm{C}_{2} \mathrm{H}_{2}$ in $\mathrm{Ar} / \mathrm{C}_{2} \mathrm{H}_{2}$ plasmas. Such a fast quenching was also experimentally observed by [32]. It results in the ionization of acetylene and brings a major contribution to the ionization kinetics and discharge equilibrium. Indeed, this quenching contributes to almost $70 \%$ of $\mathrm{C}_{2} \mathrm{H}_{2}^{+}$production and is therefore more important than the direct ionization route. The Ar* density predicted by the present model are comparable to the value measured by Stefanovic et al. [32] who found $\mathrm{Ar}^{*}$ density of $4 \times 10^{15} \mathrm{~m}^{-3}$ for a $\approx 6 \% \mathrm{C}_{2} \mathrm{H}_{2}$ feed gas. These authors also measured more than one order of magnitude decrease of $\mathrm{Ar}^{*}$ density between $\mathrm{Ar} / \mathrm{C}_{2} \mathrm{H}_{2}$ and pristine Ar plasma. This trend is consistent with the one obtained from this study although the discharge conditions simulated in this work and investigated in [32] are different.

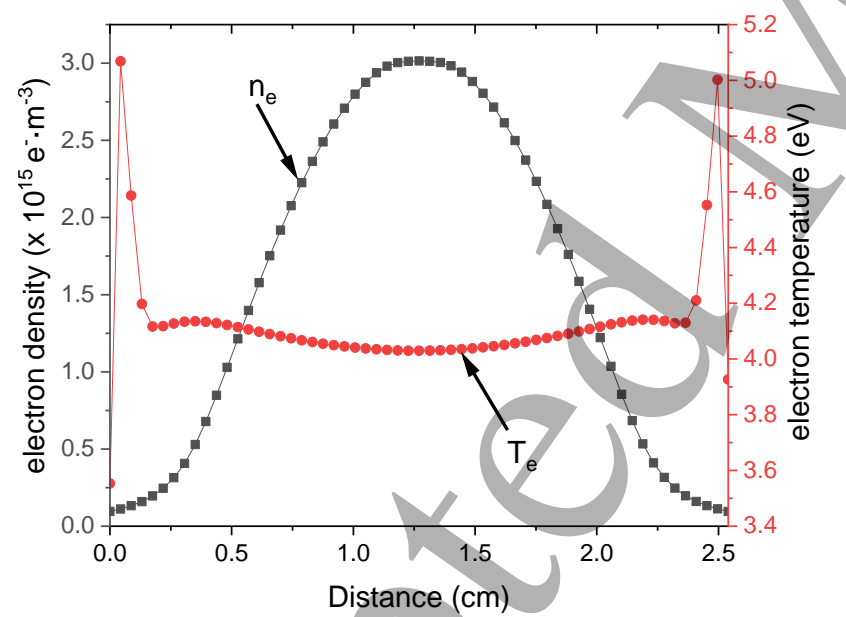

Figure 2: electron averaged density and temperature over an RF period along the discharge for convective boundary conditions

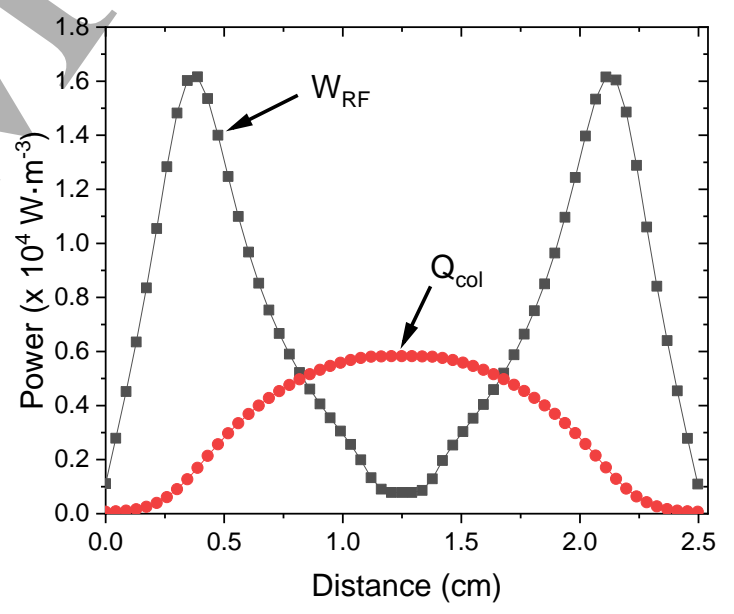

Figure 3: power density transmitted to the electron, $\mathrm{W}_{\mathrm{RF}}$ and transmitted by the electron to the plasma chemistry, $Q_{\text {coll }}$. for convective boundary conditions 


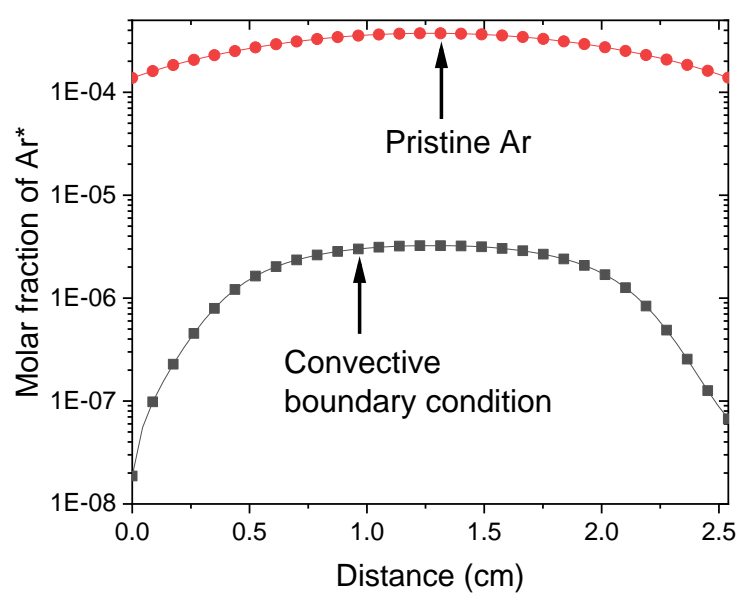

Figure 4 : Argon metastables molar fraction along the discharge in $A r / C_{2} H_{2}$ and pristine Argon discharges.

Figure5a shows the axial profiles of the major neutral species. These are $\mathrm{C}_{2} \mathrm{H}_{2}, \mathrm{C}_{2} \mathrm{H}, \mathrm{H}_{2}$ and large hydrocarbon species that have been lumped and denoted $\mathrm{C}_{x \geq 4} \mathrm{H}_{\mathrm{y}}$. It appears that, with the adopted boundary conditions, $\mathrm{C}_{2} \mathrm{H}_{2}$ mole fraction which is set to $4 \times 10^{-2}$ at the showerhead electrode, $\mathrm{x}=0 \mathrm{~cm}$, shows only slight decrease along the discharge reaching $3.83 \times 10^{-2}$ at the right electrode, which corresponds to dissociation rate of $4.3 \% . \mathrm{C}_{2} \mathrm{H}_{2}$ remains therefore the main hydrocarbon species. The conversion of acetylene is the first stage of a molecular growth chemistry that ends up with the formation of large hydrocarbon species. The total mole fraction of $\mathrm{C}_{x \geq 4} \mathrm{H}_{\mathrm{y}}$ species reaches indeed a value of $5 \times 10^{-4}$ at the right electrode. This is more than one order of magnitude greater than the mole fraction of $\mathrm{C}_{2} \mathrm{H}$ which is the main product of acetylene conversion. This clearly shows that once produced, $\mathrm{C}_{2} \mathrm{H}$-radical is rapidly involved in molecular growth processes of the type [37] : $\mathrm{C}_{2} \mathrm{H}+\mathrm{C}_{n} \mathrm{H}_{2}$ $\rightarrow \mathrm{C}_{\mathrm{n}+2} \mathrm{H}_{2}+\mathrm{H}$.

$\mathrm{C}_{2} \mathrm{H}$ radical is produced at $29 \%$ by electron-impact dissociation of acetylene, but its production is strongly enhanced by the $A r^{*}$ quenching on $\mathrm{C}_{2} \mathrm{H}_{2}$ which represents $71 \%$ of the production rate of $\mathrm{C}_{2} \mathrm{H}$ radical. This effective production of large hydrocarbon species in $\mathrm{Ar} / \mathrm{C}_{2} \mathrm{H}_{2}$ was also observed experimentally by mass spectrometry [24] and the major role of Ar metastable was experimentally shown by Stefanovic et al [32]. The strong interaction between $\mathrm{Ar}^{*}$ and molecular precursors has also been observed in Ar/HMDSO system [66]. A collisional radiative model developed by Garofano et al [66] emphasized the impact of the different precursors on the quenching of argon $1 \mathrm{~s}$ and $2 p$ states.

Figure $5 \mathrm{~b}$ shows the detail of the large $\mathrm{HC}$ molar fractions. Although the abundance decreases with the carbon number, large $\mathrm{HC}$ show significant molar fractions, which is consistent with an effective molecular growth process. The major large $\mathrm{HC}$ are in the form of $\mathrm{C}_{\mathrm{x} \geq 4} \mathrm{H}_{2}$ as it was observed in mass spectra of [24].

The other major neutral species are $\mathrm{C}_{2} \mathrm{H}$ and molecular hydrogen that shows mole fraction of about $10^{-4}$ at the stagnation electrode. The difference in the boundary conditions specified at the two electrodes results in non-symmetrical axial profiles for the neutral species. Except in the case of acetylene, the neutral species show large depletions in the vicinity of the showerhead electrode and almost no variations in the vicinity of the stagnation electrode. The almost flat mole-fraction profiles in the vicinity of the stagnation electrode show that diffusion/convection transport is much faster than surface chemistry for all the neutral species. In fact, under the investigated conditions, their transport is dominated by diffusion, i.e., their Peclet numbers are fairly small. Therefore the convective boundary condition induces a back-diffusion from the bulk of the plasma where these 
neutral species are generated to the showerhead electrode where their mole-fractions are constrained to zero. One of the major consequences of this situation is that the species loss is much more important at the showerhead electrode, i.e., the back-diffusion fluxes are dominant due to the large gradients at the showerhead electrode as compared to the stagnation electrode.
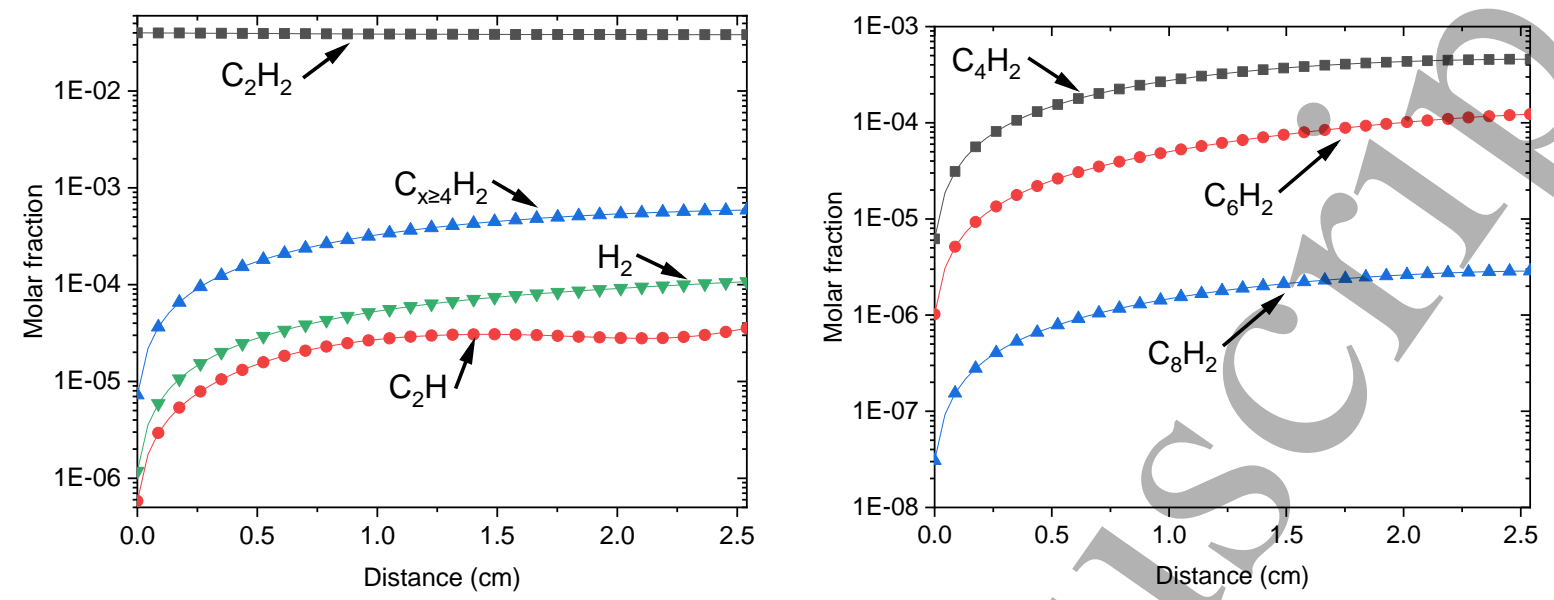

Figure 5 : distribution of (a) $\mathrm{H}_{2}, \mathrm{C}_{2} \mathrm{H}, \mathrm{C}_{2} \mathrm{H}_{2}$, and large hydrocarbons (lumped for $\mathrm{x}=4,6,8$ ) and $(\mathrm{b}) \mathrm{C}_{4} \mathrm{H}_{2}, \mathrm{C}_{6} \mathrm{H}_{2}$ and $\mathrm{C}_{8} \mathrm{H}_{2}$ along the discharge in $\mathrm{Ar} / \mathrm{C}_{2} \mathrm{H}_{2}$ discharge. for convective boundary conditions

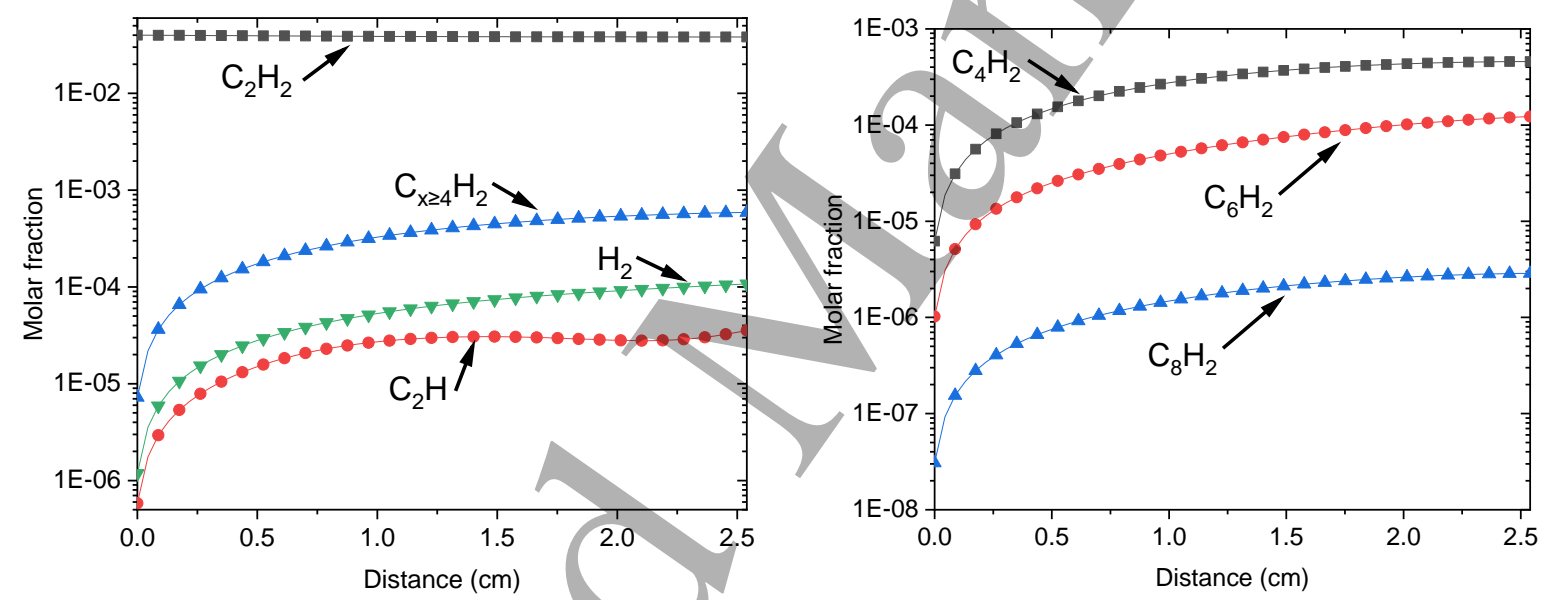

Figure 6 : distribution of (a) $\mathrm{H}_{2}, \mathrm{C}_{2} \mathrm{H}, \mathrm{C}_{2} \mathrm{H}_{2}$, and large hydrocarbons (lumped for $x=4,6,8$ ) and (b) $\mathrm{C}_{4} \mathrm{H}_{2}, \mathrm{C}_{6} \mathrm{H}_{2}$ and $\mathrm{C}_{8} \mathrm{H}_{2}$ along the discharge in $\mathrm{Ar} / \mathrm{C}_{2} \mathrm{H}_{2}$ discharge. for convective boundary conditions

Figure $7 a$ and Figure $7 \mathrm{~b}$ show the axial profiles of the major positive and negative ions. Ions with four carbon atoms and more were lumped and only their total mole fraction was reported. More detailed information on large hydrocarbon ions may be found in Figure 8. Although argon is by far the dominant neutral species, Figure $7 a$ shows that for the present simulation conditions, $\mathrm{Ar} / \mathrm{C}_{2} \mathrm{H}_{2}$ plasmas are dominated by acetylene ions with concentrations that are two-orders of magnitude larger than that of $\mathrm{Ar}^{+}$. This is clearly the consequence of the enhanced acetylene ionization through $\mathrm{Ar}^{*}$ quenching. In fact, this ionization route requires an activation energy that corresponds to the excitation threshold of the first metastables, e. g., $11.56 \mathrm{eV}$, which is significantly smaller than the energy threshold for electron impact ionization of argon, $15.8 \mathrm{eV}$. This central role of argon metastables in the ionization kinetics of argon/acetylene plasmas has been also observed experimentally by Stefanovic et al [32]. 
Figure $7 a$ also shows that the positive hydrocarbon ions are subject to a molecular growth despite their fairly limited residence time in the discharge region. As a matter of fact, the total mole fraction of $\mathrm{C}_{\mathrm{x} z 4} \mathrm{H}_{\mathrm{y}}{ }^{+}$is only two times smaller than $\mathrm{C}_{2} \mathrm{H}_{2}{ }^{+}$mole fraction and therefore largely exceeds the concentration of argon ions in the center of the discharge. The mole fraction of these large ions remains significant in the sheaths where they are only fourfold smaller than $\mathrm{C}_{2} \mathrm{H}_{2}^{+}$mole fraction.

Figure $7 \mathrm{~b}$ shows that the negative ions are strongly confined over no more than $5 \mathrm{~mm}$-width region in the discharge bulk and show a very sharp maximum at the center of the gap. The total negative ion density is slightly larger than the electron density and the plasma may be considered as electronegative in a very narrow $5 \mathrm{~mm}$-width region around the gap center. In this region, we also observe a large enhancement of positive mole-fraction with a significant/distortion of the corresponding axial-profiles. These profiles show prominent maxima in the electronegative narrow region (Figure 7a) so as to balance the large value of negative ions and insure the electrical neutrality in the bulk of the plasma. To the best of our knowledge, such a narrow distortion cannot be observed experimentally. However, similar local peaks have been observed in the simulation of silane dusty RF discharges [67] where the presence of high negative charges carried by solid particles trapped in the discharge enhanced the ion concentration to ensure local quasi-neutrality. This small electronegative region is of prime importance for the discharge equilibrium. This is indeed the only region where the negative-ions can be consumed through mutual neutralization with positive ions. In fact, the analysis of source and flux-divergence terms in the balance equations shows that negative ions are equally produced overall the discharge gap, transported to the discharge center where they are confined and then consumed by mutual neutralization with positive ions. Some of these processes are fairly long. For instance, the mutual neutralization and transport phenomena show characteristic times of 10 and $10^{-2} \mathrm{~ms}$, respectively. This is much larger than the RF period, which clearly shows the stiffness of the system and the interest of the solution procedure proposed in this work. This enables to solve for both the very fast discharge dynamics and the fairly slow processes. Outside the electronegative region at the center of the gap, the plasma is electropositive and the discharge equilibrium is governed by the balance between acetylene and argon ionization processes on one hand, and electron and positive ions losses at the electrodes on the other hand. Figure $7 \mathrm{~b}$ shows that the concentration of large negative ions is also significant. It is even slightly larger than that of $\mathrm{C}_{2} \mathrm{H}^{-}$. This shows that negative ions also experience molecular growth processes in the narrow electronegative region.

The axial profiles of charged species are perfectly symmetric despite the difference between the boundary conditions specified at the showerhead and the stagnation electrodes. This may be easily understood for negative ions that are confined in the center of the discharge and that are not sensitive to the nature of the boundary conditions specified at the electrodes. As for the positive ions, the density in the sheath near the electrodes is mainly governed by drift fluxes, i.e., diffusion can be neglected and ionization is very weak inside the sheath. The high drift velocity of the positive ions combined with their total recombination at the wall result in very small density-values at the electrodes. In fact, the difference in the boundary conditions results in only very slight differences between the ion densities at the two electrodes which can be hardly seen on the axial profiles shown in Figure $7 a$.

The details of the large positive hydrocarbon ions are shown in Figure 8a. It appears that the density of positive ions decreases by two orders of magnitude when the ion size increases from $x=4$ to $x \geq 8$ carbon atoms. This shows that the fairly short residence time of the positive ion does not allow an effective molecular growth of these species. The situation is different for negative ions. In this case 
significant population is obtained even for the largest size, i.e., $\mathrm{C}_{x \geq 8} \mathrm{H}^{-}$. Figure $7 \mathrm{~b}$ shows mole-fraction axial profiles for large negative ions with different sizes. It appears that $\mathrm{C}_{x \geq 8} \mathrm{H}^{-}$mole fraction remains significant and only three times smaller than $\mathrm{C}_{2} \mathrm{H}^{-}$mole-fraction.. These results are in good qualitative agreement with experimental mass spectrometry measurements by [48], [24], [44] which observed maximum amplitude for even carbon atom numbers species. Moreover the decreasing trend as function of mass was also observed. Jiménez-Redondo et al. [68] performed mass spectrometry measurements on an $\mathrm{Ar} / \mathrm{C}_{2} \mathrm{H}_{2}$ plasma at $10 \mathrm{~Pa}$ and for an electrode gap of $3 \mathrm{~cm}$, which is similar to our conditions, and reveal the same decreasing trend as function of the mass for both positive and negative ions. Their experimental work was coupled with a OD model which predicts a cation density of $8.8 \times 10^{14} \mathrm{~m}^{-3}$ that is also in good agreement with the one obtain in our simulations (i.e. $1.2 \times 10^{15} \mathrm{~m}^{-3}$ in the plasma bulk).

In the solution procedure used in this work the ions axial profiles are determined in both the discharge module and the LCT module. Our methodology would be valid only if the time averaged mole fraction calculated in the discharge module for each ion is in agreement with the mole fraction value obtained in the LCT module for the corresponding twin-ion. Figure 9a shows for illustration comparison between the axial profiles of three pair of ion/twin-ion mole-fraction. It appears that these axial profiles are in very good agreement. This validates our methodology that enables taking into account the coupling between the discharge dynamic and the long characteristic time ion kinetic and transport. Further, Figure $9 \mathrm{~b}$ shows the comparison between negative ion density distributions obtained from the only time-integration of the discharge dynamic equations over $1000 \mathrm{RF}$ period, i.e. $0.1 \mathrm{~ms}$, and those using the simulation procedure developed in this work. Approximately 20 iterations between the discharge dynamic module and the LCT module were carried out in this last case. It clearly appears that the negative ion density obtained from the only integration of the plasma dynamic equations is still evolving in the discharge gap. It shows values that are two orders of magnitude higher than those obtained when the time-averaged transport module is used. Actually, reaching a quasi-permanent regime for negative ions would require the time-integration of the discharge dynamic equations during more than $10^{5} \mathrm{KF}$ periods, which is practically unaffordable. It is also consistent with experimental findings of [69]. Such results may have a significant impact on the predicted molecular growth kinetics of the negative ions and the subsequent particle nucleation route.

(a)

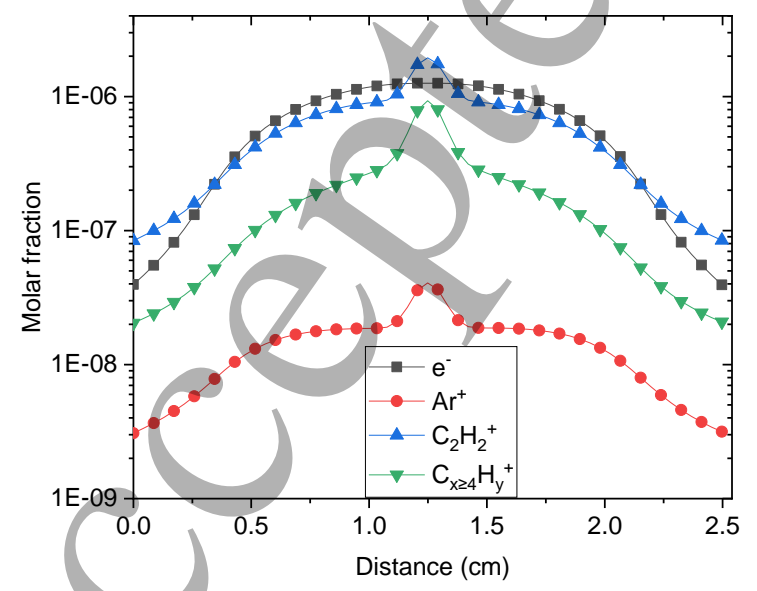

(b)

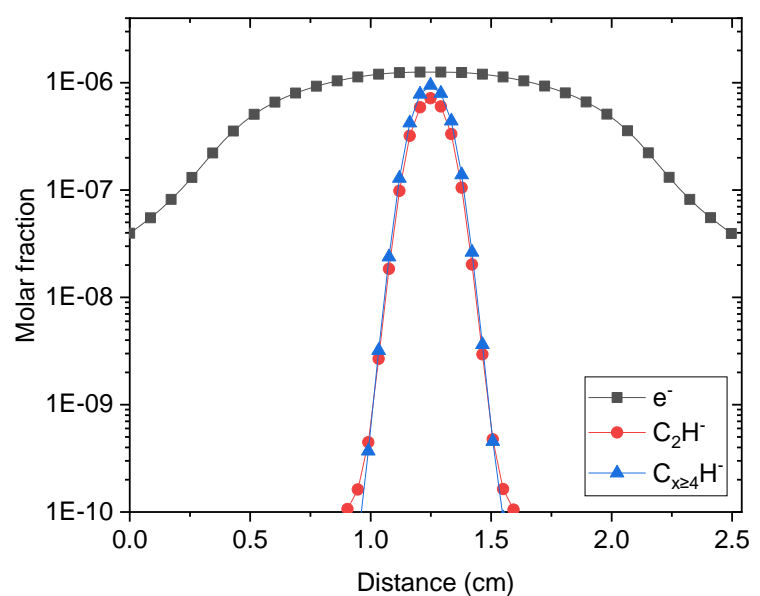

Figure 7: (a) e-, $\mathrm{Ar}+, \mathrm{C}_{2} \mathrm{H}_{2}{ }^{+}$and larger cations and (b) e-, $\mathrm{C}_{2} \mathrm{H}^{-}$and larger anions axial profiles for convective boundary 
(a)

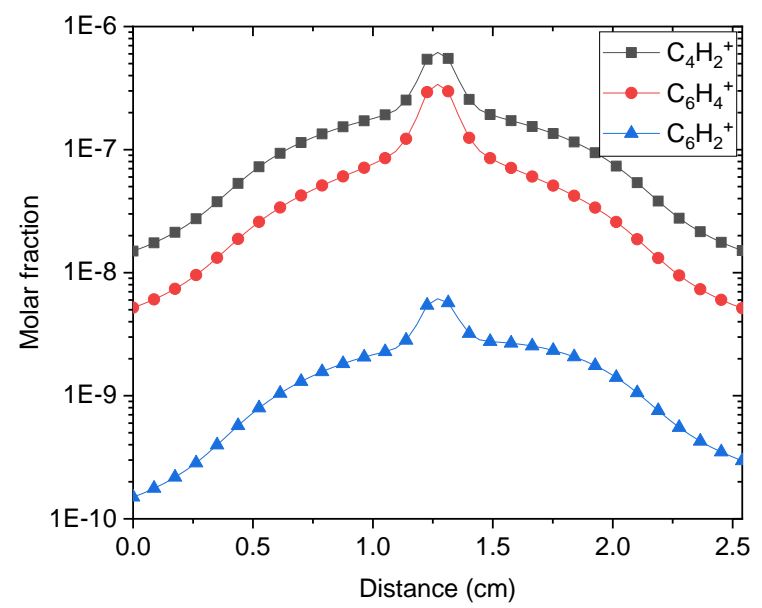

(b)

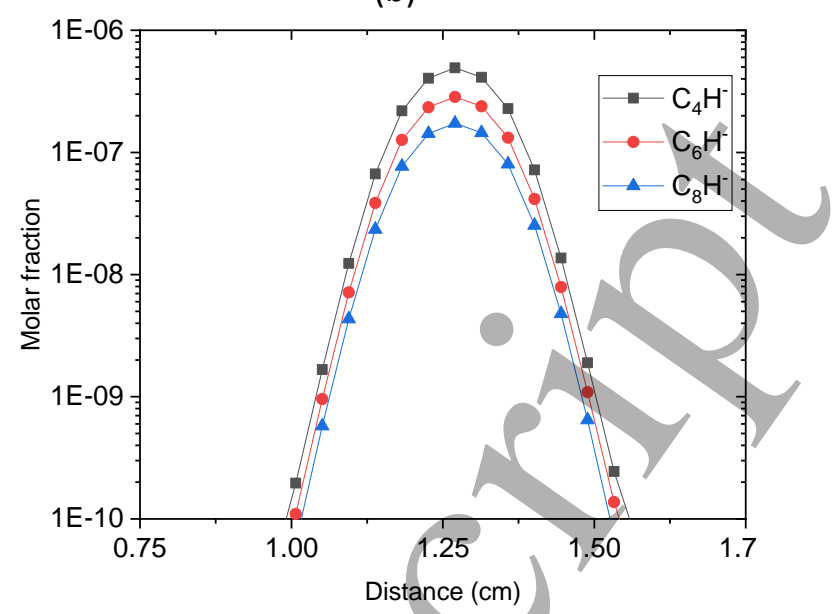

Figure 8 : Axial profiles of (a) the major positive ions and (b) the major negative ions for convective boundary conditions.

(a)

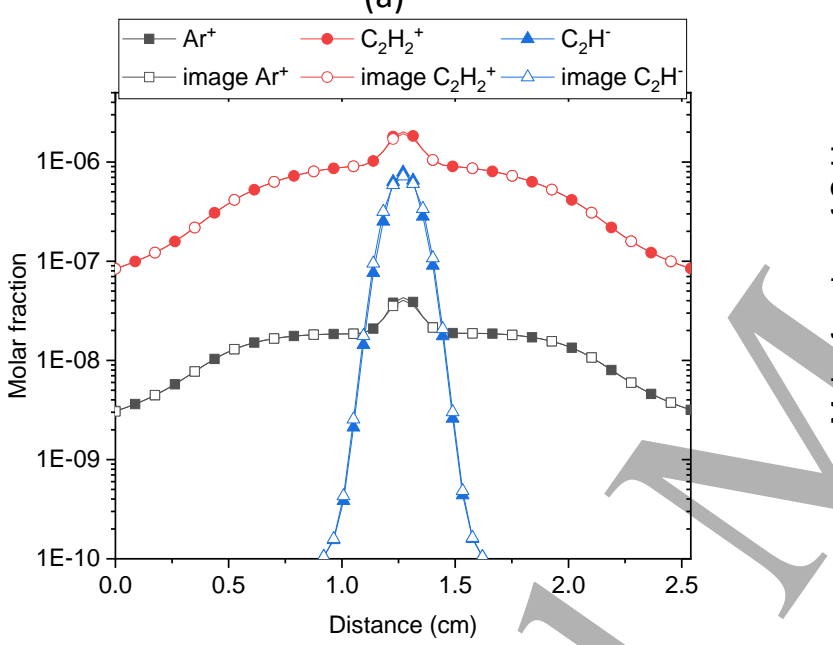

(b)

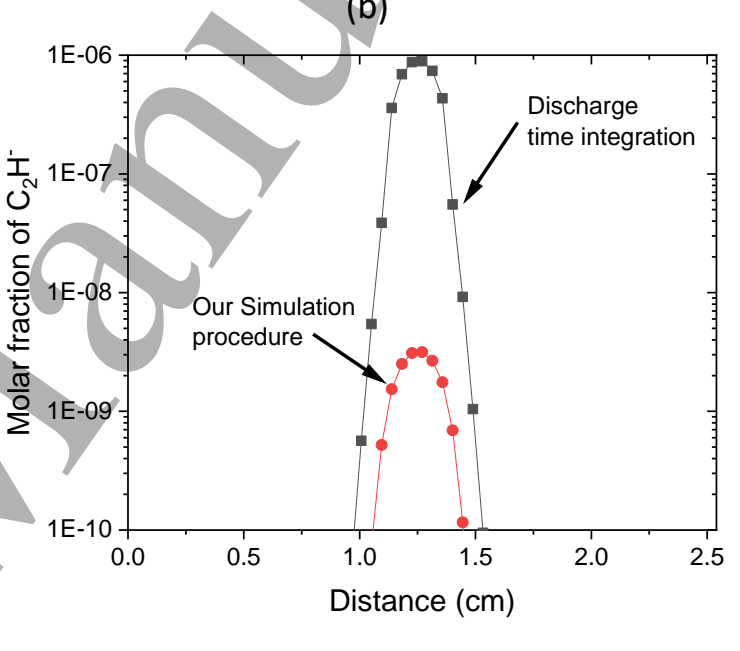

Figure 9 : Comparison of axial profiles for (a) ion and image-ion axial profiles for $\mathrm{Ar}^{+}, \mathrm{C}_{2} \mathrm{H}_{2}^{+}$and $\mathrm{C}_{2} \mathrm{H}^{-}$and (b) $\mathrm{C}_{2} \mathrm{H}^{-}$obtain after long time discharge integration and our simulation procedure.

The use of convective boundary conditions allows investigating the discharge dynamics and the plasma kinetics in the simple case where the hydrocarbon species are dominated by acetylene (the precursor species). We particularly showed that even with small acetylene concentration in the feed gas ( $4 \%$ in the present case), the central region of the discharge is electronegative, a significant molecular growth is observed on both neutral and charged species, and the ionization kinetics is dominated by quenching processes where argon metastables plays a key-role which confirms the experimental observation of Stefanovic and al [32]. Therefore we investigated the impact of the adopted values for the Penning branching ratio on the discharge composition. Using a value of 0.3 instead of 0.7 results in a decrease of $\mathrm{C}_{2} \mathrm{H}_{2}{ }^{+}$and all the positive ions by less than a factor 2 and an equivalent increase of $\mathrm{C}_{2} \mathrm{H}$. Taking into account the complexity of the model, these variations can be considered as quite limited and do not impact the observed trends and conclusions.

The simulations performed using the convective boundary conditions do not enable reproducing the large acetylene conversion that has been observed in many experiments on CCRF discharges $[24],[25]$. In fact, under the low Peclet transport conditions, imposing the feed gas composition at the showerhead electrode ensures an additional incoming diffusion flux of acetylene that is much larger 
than the convective flux generated by the gas flow. In fact, the diffusion is fast enough to insure an almost constant acetylene concentration in the plasma. Of course, this concentration that is almost the same as in the feed gas, is likely to be substantially overestimated. For this purpose, we need to use the more realistic mixed convection-diffusion-surface reaction (MCDS) boundary conditions. This will be discussed in the next sub-section.

\section{3. Mixed convection-diffusion-surface reaction boundary conditions at the showerhead electrode} - effect of gas flowrate on the discharge dynamics and plasma composition.

a) Influence of the boundary conditions on the discharge dynamics and plasma composition in the showerhead configuration

Simulations were then carried out with the MCDS boundary conditions (Equation 18) specified at the two electrodes with 5 values of $u_{\text {feed, }}$ i.e., $5 \times 10^{-2}, 0.1,0.25,0.5$ and $1 \mathrm{~m} . \mathrm{s}^{-1}$, at the showerhead electrode located at $\mathrm{x}=0 \mathrm{~m}$ and $u_{\text {elec }}=0$ at the stagnation electrode located at $\mathrm{x}=2.54 \mathrm{~cm}$.

Figure 10 show the axial profile of the electron density, the electron temperature and Ar metastable for the two types of boundary conditions applied to the neutral species at the showerhead electrode. We observe that the values of electron density and temperature obtained with MCDS boundary conditions at large flowrates, typically between 0.5 and $1 \mathrm{~m} \cdot \mathrm{s}^{-1,}$ are similar to those obtained with convective boundary condition. However, bulk value of $\mathrm{Ar}^{*}$ molar fraction is slightly smaller for convective boundary condition. This smaller Ar* molar fraction values is due to the artificially imposed constraint $\left.Y_{A r *}\right|_{\text {elec }}=0$ in the case of convective boundary conditions..

Figure 10a shows the axial profiles of the acetylene mole-fraction in the gap for the different values of the feed gas inlet velocity along with the distributions obtained with the convective boundary conditions. We observe that when MCDS physical boundary conditions are imposed, the obtained acetylene mole-fraction in the plasma is much smaller than the corresponding feed gas value, i.e. $4 \times 10^{-2}$, and also smaller than the values obtained using the convective boundary conditions. Its value in the bulk of the plasma decreases from $2 \times 10^{-2}$ for $u_{\text {feed }}=1 \mathrm{~m} \cdot \mathrm{s}^{-1}$ to $10^{-4}$ for $u_{\text {feed }}=5 \times 10^{-2} \mathrm{~m} \cdot \mathrm{s}^{-1}$. These results show that acetylene is in fact highly converted in the acetylene/argon plasma investigated

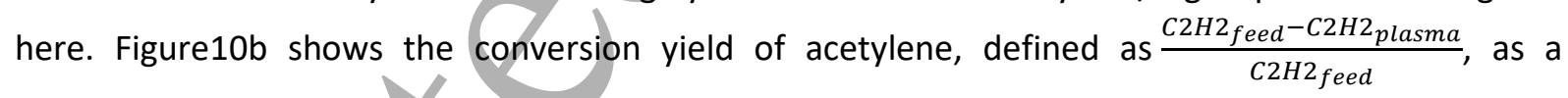
function of the inlet feed-gas flow velocity. Even with a fairly high inlet velocity-value, i.e., $u_{\text {feed }}=1$ $\mathrm{m} . \mathrm{s}^{-1}$, corresponding to very short residence time in the gap, i.e., $\tau_{\text {res }}=700 \mathrm{~ms}, 50 \%$ of acetylene is converted. The conversion yield is as high as $90 \%$ for an inlet velocity smaller than $0.35 \mathrm{~m} . \mathrm{s}^{-1}$ and becomes almost total, i.e. $\approx 100 \%$, for a flow velocity smaller than $0.25 \mathrm{~m} . \mathrm{s}^{-1}$.

Conversion yields greater than 90\% were also measured in [32], [24],[25] for acetylene in $\mathrm{Ar} / \mathrm{C}_{2} \mathrm{H}_{2}$ plasmas. Such total conversion yields were also predicted in the plasma bulk using a global $\mathrm{OD}$ modeling by Denysenko et al [44] and by Ariskin et al. [43] who used a hybrid approach. Such large conversion yields of the precursor are also consistent with many experimental results observed in other Ar/molecular precursor systems such as Ar/HMDSO [66].

The acetylene mole-fraction shows very small space variation in the gap except in the case of very small inlet velocity values. This means that diffusion transport is fast enough to induce an effective 
A close look to 10 reveals that the bulk-values for electron density, electron temperature and argon metastables density exhibit two slight variation domains at large and low inlet velocity separated by a sharp transition around $0.25 \mathrm{~m} . \mathrm{s}^{-1}$. For instance, the bulk electron mole-fraction shows less than $10 \%$ relative variation in the inlet velocity ranges $0.5-1 \mathrm{~m} . \mathrm{s}^{-1}$, a sharp threefold decrease in the velocity range $0.1-0.5 \mathrm{~ms}^{-1}$ and around $20 \%$ relative variations in the velocity range 0.05-0.1 m.s. Actually, this behavior corresponds to a transition between two ionization regimes (cf. Figure 10). In the first one, obtained for a gas flow velocity in the range 0.25-1 m.s. ${ }^{-1}$, the Penning ionization of $\mathrm{C}_{2} \mathrm{H}_{2}$ by $\mathrm{Ar}$ * strongly dominates the ionization kinetics, which leads to an enhanced production of $\mathrm{C}_{2} \mathrm{H}_{2}{ }^{+}$, and the discharge is largely dominated by hydrocarbon ions. In the second regime obtained for inlet flow velocity below $0.25 \mathrm{~m} . \mathrm{s}^{-1}$, electron impact ionization of $\mathrm{Ar}^{*}$ becomes dominant and leads to an enhanced production of $\mathrm{Ar}^{+}$. Figure 10 shows that this transition corresponds to more than two orders of magnitude variations in the ionization frequencies of the above mentioned ionization routes. Further, while the increase of the frequency of Penning ionization of $\mathrm{C}_{2} \mathrm{H}_{2}$ by $A r^{*}$ is directly linked to the increase in the acetylene density (cf. Figure 11), the frequency of ionization by electron impact on $\mathrm{Ar}^{*}$ is correlated to the change of electron density and temperature, i.e., $v_{A r^{*}-e}=k_{i-A r^{*}}\left(T_{e}\right) n_{e}$. It appears that the change in electron impact ionization frequency with the inlet flow velocity is determined by the electron temperature variation. As a matter of fact, both electron-temperature

mixing and an almost uniform acetylene concentration. In fact, this fast diffusion explains the conditions. In the case of MCDS boundary conditions, the constraint on the acetylene concentration at the showerhead electrode is relaxed and the overall acetylene concentration in the feed gas is determined by the balance between the amounts of acetylene introduced into the reactor and converted in the discharge. As a consequence, acetylene mole fraction at the showerhead electrode is much smaller than the feed gas values since acetylene is highly converted in the bulk and $\mathrm{C}_{2} \mathrm{H}_{2}$ diffuses from the inlet to the central region where $\mathrm{C}_{2} \mathrm{H}_{2}$ is converted. Therefore, the application of MCDS physical boundary conditions is a absolutely required for a satisfactory description of discharge conditions with large acetylene conversion yields.

\section{B ) Influence of the flowrate on the discharge dynamics and plasma composition in the} showerhead configuration

One can deduce the effect of flowrate on the electron temperature and density, argon metastable from Figure 10. It appears that the electron mole-fraction in the bulk of the plasma increases by more than a factor 4, i.e. from $0.3 \times 10^{-6}$ to $1.3 \times 10^{-6}$, when $u_{\text {feed }}$ increases from 0.05 to $1 \mathrm{~m} . \mathrm{s}^{-1}$. On the other hand, the electron-temperature shows an opposite trend and decreases by $1 \mathrm{eV}$, from $5 \mathrm{eV}$ to $4 \mathrm{eV}$, for the same flow velocity variation. The effect of $u_{\text {feed }}$ is even more pronounced on argon $5 \times 10^{-2} \mathrm{~m} \cdot \mathrm{s}^{-1}$ to $10^{-6}$ at $1 \mathrm{~m} \cdot \mathrm{s}^{-1}$. From Figure $10 \mathrm{a}$, it appears that when the flow velocity is decreased by a factor 20 , the acetylene mole-fraction in the plasma decreases by a factor 400 . The change in acetylene concentration with the flow velocity (or residence time) is therefore non-linear and most of the acetylene conversion yield variation takes place over the inlet velocity range $0.25-1 \mathrm{~m} \cdot \mathrm{s}^{-1}$. As a matter of fact, the conversion yield increases from $45 \%$ at $1 \mathrm{~m} \cdot \mathrm{s}^{-1}$ to $95 \%$ at $0.25 \mathrm{~m} . \mathrm{s}^{-1}$. Further decrease of the inlet velocity results in a very slight change of the conversion yield since acetylene is already almost totally converted at $0.25 \mathrm{~m} . \mathrm{s}^{-1}$. 
and ionization frequencies decrease with increasing $u_{\text {feed }}$, while the electron density as well as the power coupled to the discharge ( $3.6 \mathrm{~W}$ for $u_{\text {feed }}=0.05 \mathrm{~m} . \mathrm{s}^{-1}$ and $6 \mathrm{~W}$ for $u_{\text {feed }}=1 \mathrm{~m} . \mathrm{s}^{-1}$ ) increases with the same parameter. It appears therefore that the change in the discharge parameters, i.e., $n_{e}, T_{e}$ and Ar* density with $u_{\text {feed }}$ is not due to residence time effect as in the case of acetylene conversion, but rather to the change in the ionization kinetics, discharge dynamics and power coupling with the level of acetylene in the plasma.

It is worthy to mention here that the contribution of acetylene to the ionization kinetics remains significant even for flow velocity conditions leading to very small amount of acetylene. This may be clearly observed in Figure 13.a and 13.b that show the axial profiles of the major positive ion mole fractions for flow velocity values of $0.05 \mathrm{~m} . \mathrm{s}^{-1}$ and $1 \mathrm{~m} \cdot \mathrm{s}^{-1}$, respectively. Especially, $\mathrm{C}_{2} \mathrm{H}_{2}{ }^{+}$mole fraction is approximately $1.2 \times 10^{-7}$ in the bulk of the discharge, which is only 2 times lower than $\mathrm{Ar}^{+}$molefraction for $u_{\text {feed }}=5 \times 10^{-2} \mathrm{~m} \cdot \mathrm{s}^{-1}$, a condition for which the $\mathrm{C}_{2} \mathrm{H}_{2}$ fraction is $10^{-4}$. The situation is very different for $u_{\text {feed }}=1 \mathrm{~m} . \mathrm{s}^{-1}$ when acetylene fraction in the plasma remains significant. In this case, both $\mathrm{C}_{2} \mathrm{H}_{2}{ }^{+}$and large hydrocarbon ions dominate the discharge, while argon ion fraction is more than one order of magnitude smaller. The large hydrocarbon positive ions are much more sensitive to the flow velocity than $\mathrm{C}_{2} \mathrm{H}_{2}{ }^{+}$. The population of these ions increases with $\mu_{\text {feed }}$ and varies by more than three orders of magnitude in the investigated range of flow velocity. The $\mathrm{HC}$ positive ion abundances obtained in this study present some discrepancies with the previously simulated ion densities [44] and [43]. As a matter of fact Ariskin et al. [43] predicted a much larger $\mathrm{Ar}^{+}$and large $\mathrm{HC}$ ion densities. We believe that the difference with our results is due to the fact that the ionization of HC species through $\mathrm{Ar}^{*}$ was not considered in [43], while these processes represent the main ionization route in our conditions. Denysenko et al. [44] obtained $\mathrm{C}_{4} \mathrm{H}_{2}{ }^{+}$as the major ion which is also different from the prediction of our model. This may be explained by the difficulty of evaluating ion fluxes at the electrodes in a global model which may impact the rate of surface losses which are the major consumption term in the balance equation of positive ions in our model. Note however that our ion densities distribution at high flowrate are fully consistent with the ions mass spectrum presented in [44] as $\mathrm{C}_{2} \mathrm{H}_{2}^{+}$is the major specjes, followed by large $\mathrm{HC}$ ions and a much smaller $\mathrm{Ar}^{+}$relative abundance.

As for negative ions shown in Figure 13.c and 13.d, flow conditions leading to larger acetylene amount in the plasma, e.g., $u_{\text {feed }}=1 \mathrm{~m} . \mathrm{s}^{-1}$, results in an enhanced attachment kinetics and an almost two orders of magnitude larger negative ions concentration. As a result, the plasma shows a significant electronegativity for large flow velocity conditions. In this case the discharge structure is similar to the one obtained with the convective boundary conditions discussed in the previous section with a very narrow, i.e., $5 \mathrm{~mm}$ width, electronegative region in the plasma bulk surrounded by a much larger electropositive region. Note however that, as in the case of positive ions, the concentration of negative ions remains significant and almost equal to the electron concentration in the bulk of the discharge even for low flow rate conditions when the acetylene fraction is as low as $10^{-4}$. The evolutions of the negative ion density with the ion size obtained in our model show the same trends as those reported in [45] and [43]. However, our model predicts larger negative ion density, i.e. at least one order of magnitude, and a higher electronegativity in the very center of the discharge gap. It is very difficult to explain such differences. A possible explanation would be the differences in (i) the gap distance (ii) the attachment kinetics (attachment on large molecules) and/or (iii) the prediction of the long characteristic time negative ion transport dynamics and mutual recombination. 
Beside acetylene, the major neutral hydrocarbon species obtained in the discharge are $\mathrm{C}_{2} \mathrm{H}$-radical and large $\mathrm{C}_{x \geq 4} \mathrm{H}_{y}$ species, the axial distributions of which are depicted in Figure 14. The concentration of $\mathrm{C}_{2} \mathrm{H}$ radical is only slightly affected by the gas flow velocity. Basically, although its axial profile shows significant variation, the bulk $\mathrm{C}_{2} \mathrm{H}$ concentration shows the same order of magnitude, $\approx 10^{-5}$, for all the investigated flow velocity values. On the opposite, the production of large neutral hydrocarbons is strongly enhanced for large flow velocities and the $\mathrm{C}_{\mathrm{x} \geq 4} \mathrm{H}_{\mathrm{y}}$ fraction increases from $10^{-6}$ to $3 \times 10^{-3}$ with $u_{\text {feed }}$ in the investigated range of this parameter. We note however that $\mathrm{H}_{2}$ molefraction is almost constant, of the order of $10^{-2}$, for all the investigated flow conditions. Molecular hydrogen may become the major neutral species in the discharge. It is indeed produced by $\mathrm{C}_{2} \mathrm{H}_{2}$ conversion as well as deposition and molecular growth processes. Although each of these processes changes with $u_{\text {feed, }}$, the balance of the overall production rate of $\mathrm{H}_{2}$ remains almost constant in the investigated conditions. At large flowrate these results are consistent with the mass spectrum measured by [44].

The results obtained show that large flow rate conditions yield much greater densities of charged and neutral large hydrocarbons, which would indicate that nucleation kinetics should be enhanced for the flow velocity range investigated in this work.

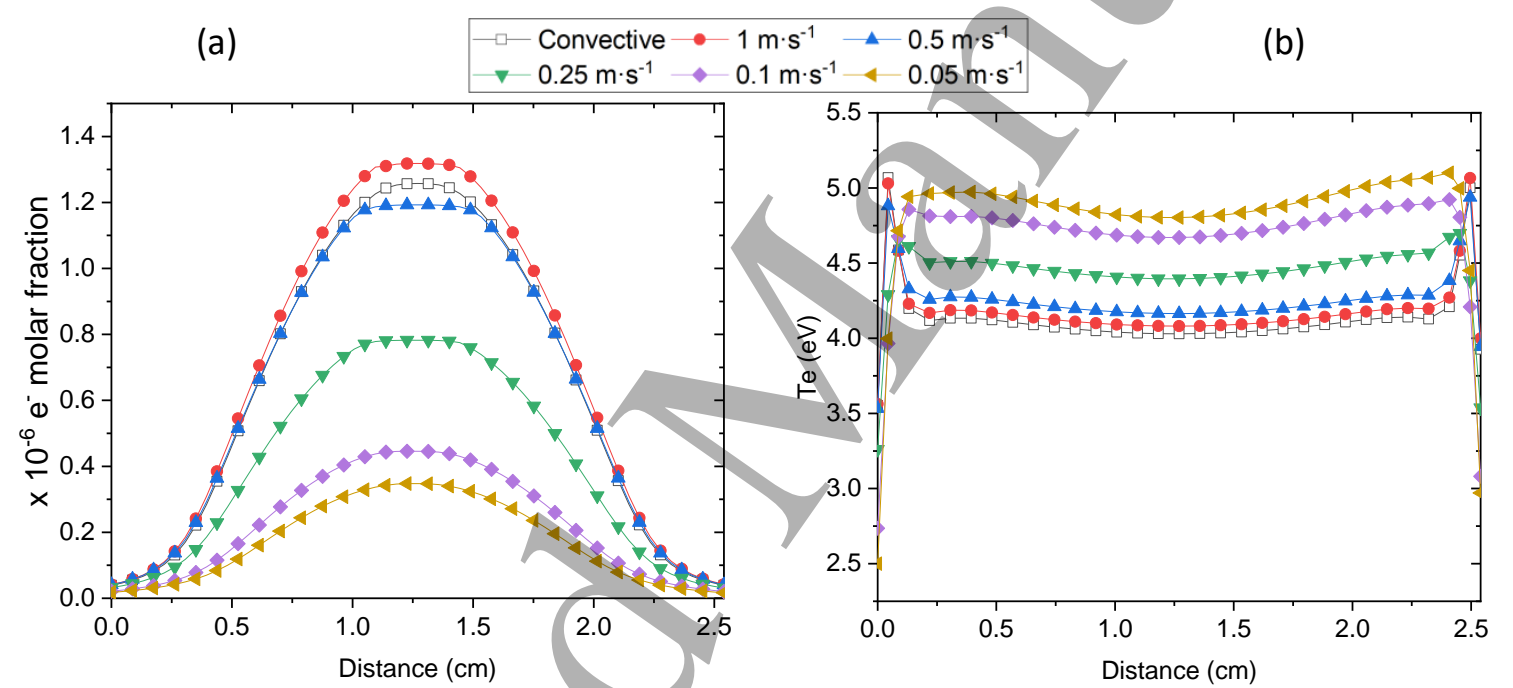

(c)

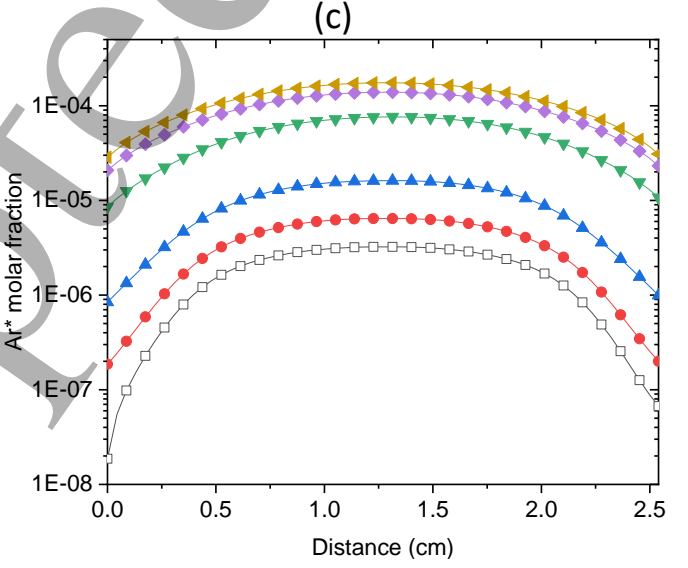

Figure 10: Axial profiles of electron mole fraction (a), electron-temperature (b) and argon-metastables. (c) in the gap for different inlet flow velocity-values. 
(a)

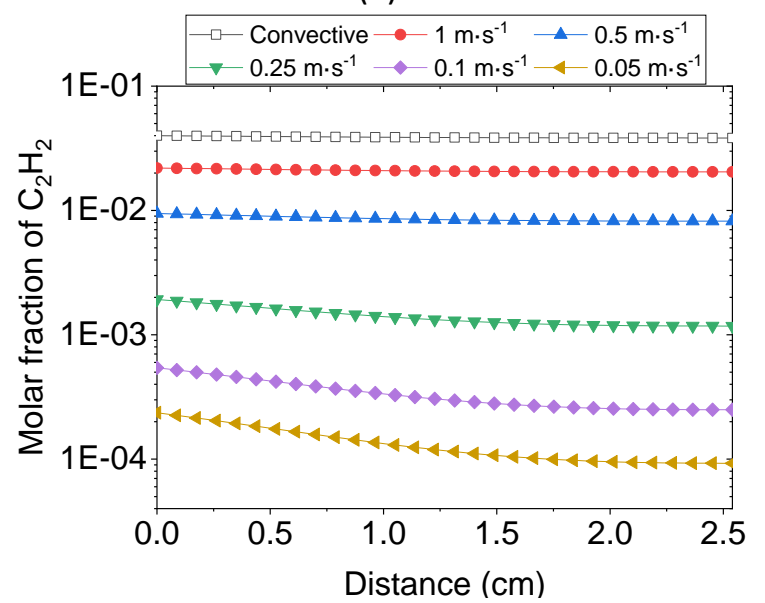

(b)

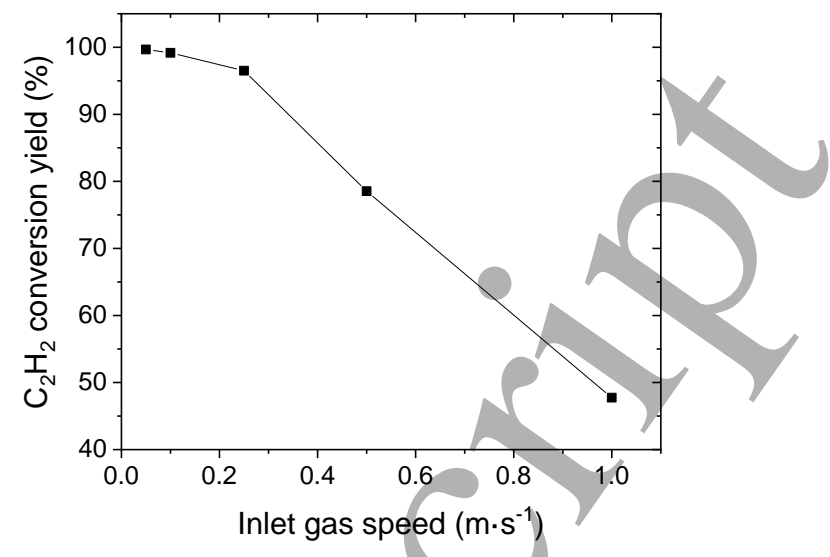

Figure 11: (a) Axial profile of acetylene mole fraction in the gap for convective (blank square) and MCDS (solid symbol) boundary conditions with different inlet flow velocity-values and (b) corresponding acetylene conversion yield as a function of the inlet flow velocity.

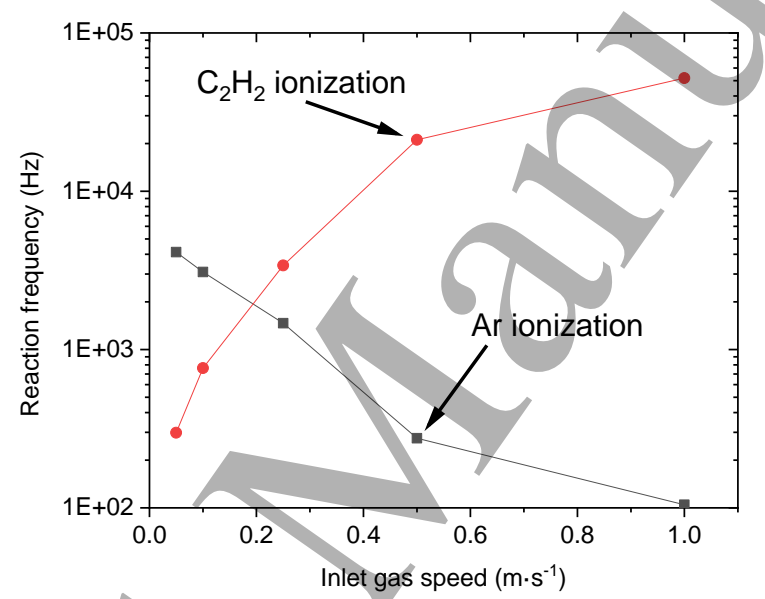

Figure 10 frequency of Penning ionization of $\mathrm{C}_{2} \mathrm{H}_{2}$ by $\mathrm{Ar}$ * and electron-impact on $\mathrm{Ar}$ * as function of inlet flow velocity value in MCDS boundary conditions. 


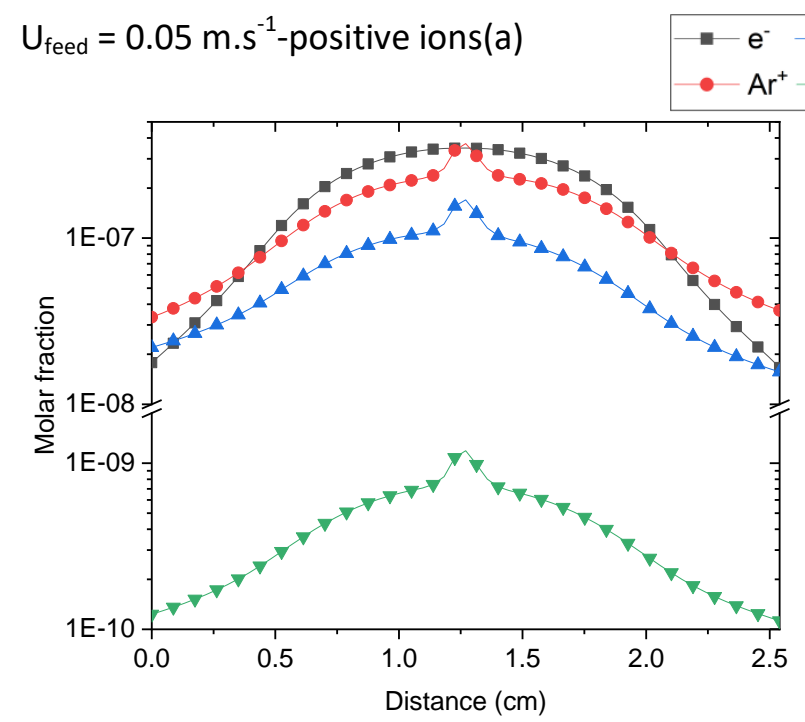

$\mathrm{U}_{\text {feed }}=0.05 \mathrm{~m} . \mathrm{s}^{-1}$-negative ions (c) $\because \mathrm{e}^{-} \longrightarrow \mathrm{C}_{2} \mathrm{H}^{-} \triangle \mathrm{C}_{\mathrm{x} \geq 4} \mathrm{H}^{-} \quad \mathrm{U}_{\text {feed }}=1 \mathrm{~m} \cdot \mathrm{s}^{-1}$-negative ions (d)

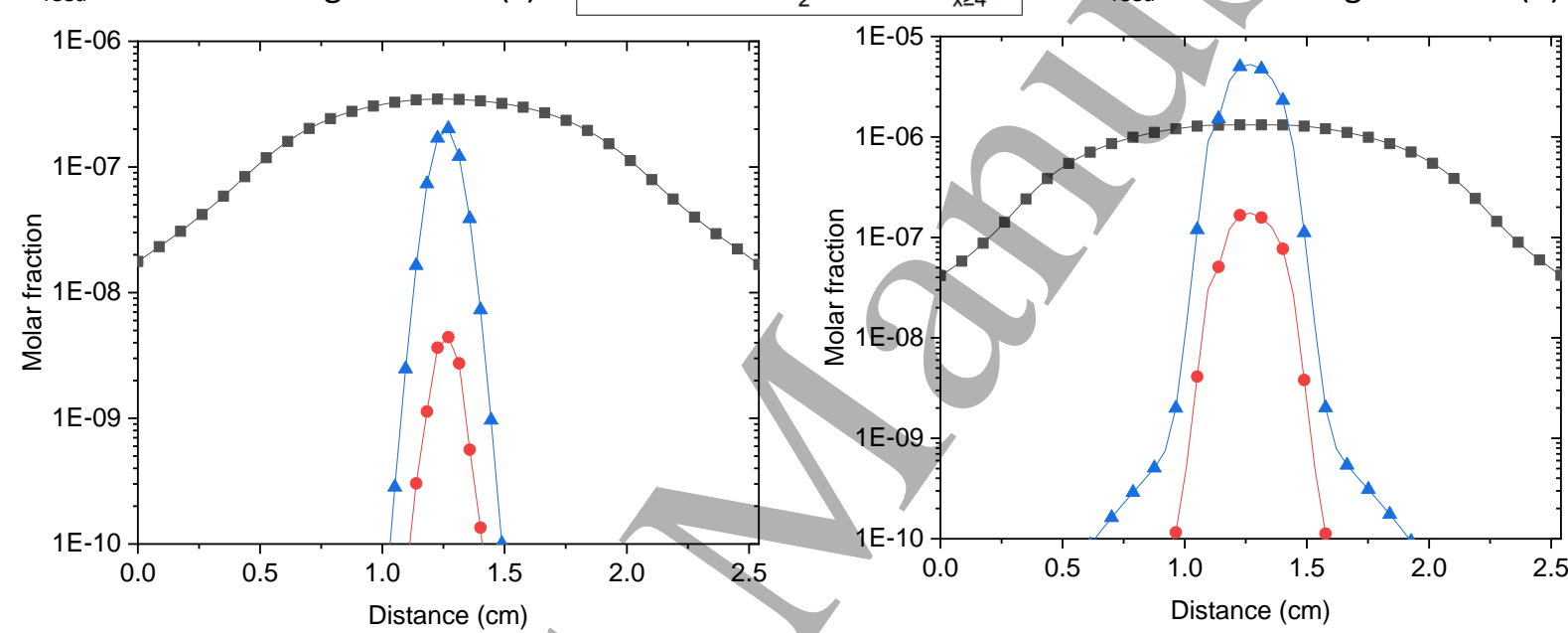

Figure 13: axial profiles of the major positive and negative ions in the discharge gap for the two discharge regimes using MCDS boundary conditions with inlet flow velocity of $5 \times 10^{-2} \mathrm{~m} \cdot \mathrm{s}^{-1}$ and $1 \mathrm{~m} \cdot \mathrm{s}^{-1}$

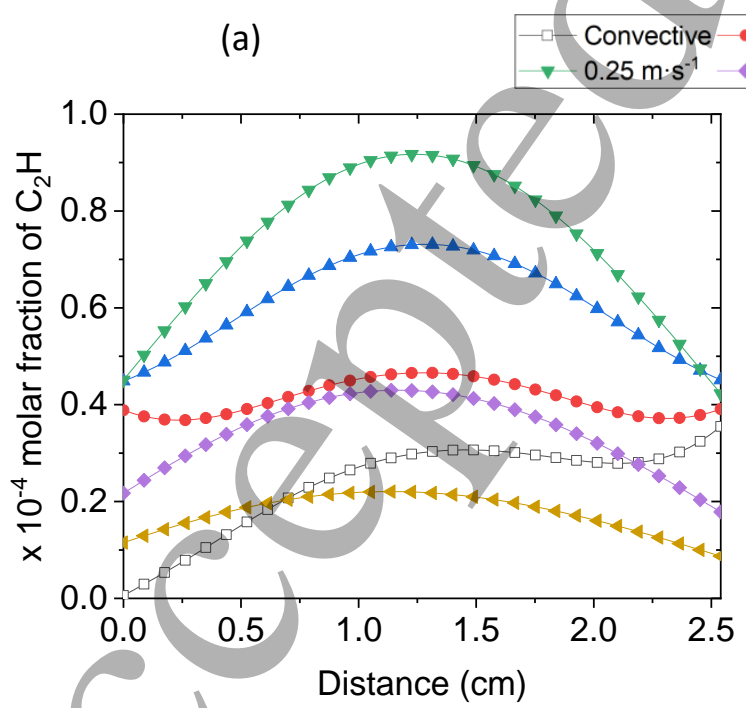

$1 \mathrm{~m} \cdot \mathrm{s}^{-1}-0.5 \mathrm{~m} \cdot \mathrm{s}^{-1}$
$0.1 \mathrm{~m} \cdot \mathrm{s}^{-1}-0.05 \mathrm{~m} \cdot \mathrm{s}^{-1}$

(b)

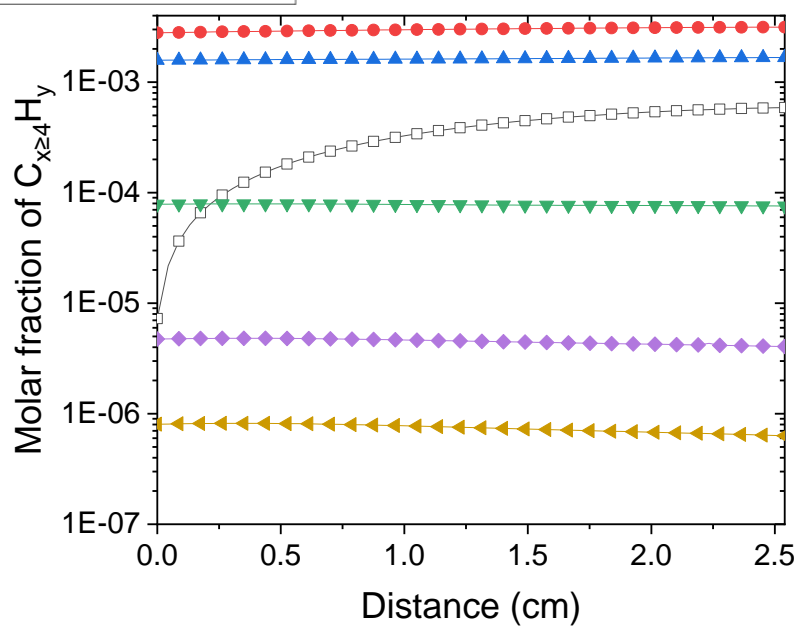

Figure 14: $\mathrm{C}_{2} \mathrm{H}$ radical(a) and large hydrocarbon(b), i.e., $\mathrm{C}_{\mathrm{x} 22} \mathrm{H}_{\mathrm{y}}$, mole-fraction in the gap for convective (blank square) and MCDS (solid symbol) boundary conditions with different inlet flow velocity-values 


\section{4. Effect of the flow configuration on the discharge dynamics and the plasma composition: comparison between showerhead and remote feed gas systems}

In this section we compare the plasma composition and discharge dynamics obtained for the showerhead feed gas inlet discussed in the previous section and the remote feed gas configurations. As discussed in section II. 3., we propose to analyze the plasmas obtained for two asymptotic situations. The first one where $\delta=\frac{V_{\text {gap }}}{V_{\text {reactor }}} \rightarrow 1$ corresponds to a situation where the discharge gap is directly and homogeneously fed by the fresh gas while the plasma products exit the discharge gap with the gas flow. The second case, where $\delta \rightarrow 0$, corresponds to a full dilution of the discharge products in the reactor volume that shows the same composition as the feed gas. In both situations the flow effect appears as a source term in the species equations. This source term does not depend on the flow rate when the dilution is important, i.e., $\delta \rightarrow 0$. In this case, the flow effect is formulated as a mass transfer phenomenon between the discharge gap and the surrounding post-discharge reactor volume. Therefore, only a single simulation is performed for this case. For the $\delta \rightarrow 1$ case, we performed two simulations $\delta \rightarrow 1 @ 10 \mathrm{sccm}$ and $\delta \rightarrow 1 @ 100 \mathrm{sccm}$. These two values of the flowrate correspond to flow inlet velocities of $0.05 \mathrm{~m} . \mathrm{s}^{-1}$ and $0.5 \mathrm{~m} . \mathrm{s}^{-1}$ in the case of the showerhead configuration.

Figure 15.a and 15.b show the axial profiles of the major neutral species. As in the case of the showerhead configuration, smaller flowrate results in an enhanced acetylene conversion. The acetylene mole fraction decreases by more than two orders of magnitude when the flowrate is decreased from $100 \mathrm{sccm}$ to $10 \mathrm{sccm}$. This trends is fully consistent with the results obtain for the showerhead configuration, although a factor two difference exists in the absolute mole fraction of acetylene between the two configurations for the large flowrate case. The increase of acetylene conversion for small flowrates does not benefit to the formation of the other hydrocarbon species. For instance Figure 15.a and 15.b show that both $\mathrm{C}_{2} \mathrm{H}_{2}$ and $\mathrm{C}_{x>4} \mathrm{H}_{y}$ mole fractions increase with the flowrate. The mole fraction variations for the large hydrocarbon molecules are of the same order of magnitude as for acetylene and much more significant than for $\mathrm{C}_{2} \mathrm{H}$ radical. It appears therefore that at low flowrate, the major fraction of $\mathrm{C}_{2} \mathrm{H}$ radicals produced by the enhanced $\mathrm{C}_{2} \mathrm{H}_{2}$ dissociation undergo surface reaction, and deposition is favored over molecular growth (which is a second order process). This trend is also consistent with the results obtained with the showerhead configuration.

The most striking difference between the two studied configurations is observed on molecular hydrogen mole fraction for large flowrates. As a matter of fact, while $\mathrm{H}_{2}$ fraction remains around $10^{-2}$ for showerhead configuration, values as high as 0.1 are obtained for remote feed gas inlet configuration at $100 \mathrm{sccm}$. To understand this difference the mass balance of hydrogen needs to be considered. Hydrogen is produced by acetylene conversion in the discharge gap and is mainly lost with the discharge products out-flowing the discharge gap. While the production rates of $\mathrm{H}_{2}$ are similar, hydrogen loss terms show a large difference between the two configurations. As a matter of fact, the rate of the radial convective losses in the stagnation line theory, i.e., the term $\rho Y_{s} \frac{d u}{d x}$ in equation 15, results in much larger flow induced loss rate values than those estimated on the basis of a uniform gas flow exiting the discharge volume, i.e., the term $\frac{Q_{m}^{\text {out }} Y_{S}}{V_{\text {plasma }}}$ in equation 17 , used in the remote feed gas configuration with $\delta \rightarrow 1$.

Although the acetylene inlet fluxes used in the $\delta \rightarrow 0$ and $\delta \rightarrow 1 @ 100 \mathrm{sccm}$ cases are pretty similar, the $\mathrm{C}_{2} \mathrm{H}_{2}$ mole-fraction obtained at large dilution, i.e., $\delta \rightarrow 0$, is one order of magnitude smaller (Figure 
15c) which indicates that the acetylene conversion is greater. This is related to the large difference between the hydrogen mole fraction values obtained in the two cases, i.e., $10^{-3}$ and $10^{-1}$, respectively. As a matter of fact, the much lower hydrogen content in the $\delta \rightarrow 0$ case results in a smaller energy dissipation, a higher electron density, an enhanced $A r^{*}$ production and a subsequent larger $\mathrm{C}_{2} \mathrm{H}_{2}$ conversion. Note that as mentioned previously, this difference in molecular hydrogen contents is due to the difference between the rates of hydrogen loss with the gap outflow in the two models. In any case, this shows that large dilutions, i.e., small discharge gap to reactor volume ratios, or large flowrates lead to acetylene dominated ionization kinetics with $X_{\mathrm{C}_{2} \mathrm{H} 2}$ typically above $10^{-3}$. On the opposite small dilutions and flowrates result in argon dominated ionization kinetics and very small acetylene concentrations, typically $\mathrm{X}_{\mathrm{C} 2 \mathrm{H} 2} \approx 10^{-4}$. The large hydrocarbons follow the same trends as acetylene while small radicals, such as $\mathrm{C}_{2} \mathrm{H}$, shows fairly moderate variations as function of the flowrate or the discharge to the reactor volume ratios.

This change in acetylene fraction has a strong effect on the discharge dynamics, as well as the ionization and attachment kinetics. When decreasing the flowrate, the discharge dynamic transitions from a situation where charged species are dominated by $\mathrm{C}_{2} \mathrm{H}_{2}^{+}$and $\mathrm{C}_{x \geq 4} \mathrm{H}_{y}^{+}$and where the attachment is significant to a situation where the dominant ion is $\mathrm{Ar}^{+}$and the attachment is negligible. This may be clearly seen in Figure 16 and Figure 17 that show the axial profiles of positiveion and negative-ion mole-fractions, for the three cases discussed in this section. As in the case of the showerhead electrode, an enhanced ionization with larger electron mole-fraction (a factor of 2) and significantly smaller electron temperature $\left(\delta T_{e}\right.$ of $\left.1 \mathrm{eV}\right)$ is observed at large flow rate (b) and for large dilution (c), i.e., $\delta \rightarrow 0$. In these cases the discharge is strongly electronegative in a localized region around the mid-gap (Figures 16b, 16.c 17.b and 17.c). The electronegativity in this region is insured by the large hydrocarbon negative ions that show a large density, a factor 5-8 larger than electron density and two orders of magnitude larger than the $\mathrm{C}_{2} \mathrm{H}^{-}$density (Figures 17.b and 17.c). The situation is different for the positive ions since although large hydrocarbon ions are significant, $\mathrm{C}_{2} \mathrm{H}_{2}{ }^{+}$ also shows large densities (Figure 16.b and 16.c) and even becomes the major ion with densities that exceed those of $\mathrm{C}_{x \geq 4} \mathrm{H}_{y}{ }^{+}$by one order of magnitude for large dilution, i.e., $\delta \rightarrow 0$ (Figure 16.c). As in the case of the showerhead configuration, the plasma is electropositive and dominated by hydrocarbon ions outside this narrow electronegative region, i.e., both $\mathrm{C}_{2} \mathrm{H}_{2}{ }^{+}$and large $\mathrm{HC}$ in the case $\delta \rightarrow 1 @ 100$ sccm and $\mathrm{C}_{2} \mathrm{H}_{2}{ }^{+}$for $\delta \rightarrow 0$ (Figure 16.b and 16.c and Figure 17.B and 17.c). For small flow rates, i.e., 10 sccm, the discharge is electropositive and dominated by argon ions, although the population of $\mathrm{C}_{2} \mathrm{H}_{2}{ }^{+}$ remains significant and only a factor 2 below $\mathrm{Ar}^{+}$population. This result is quite remarkable: the contribution of acetylene to the ionization kinetics remains highly significant despite its very low concentration, i.e., $10^{-4}$, in the discharge. In fact acetylene depletion at low flowrate has much more impact on the large hydrocarbon ions, the concentration of which decreases by more than three orders of magnitude when acetylene is depleted. On the opposite, even though the discharge becomes electropositive, the large negative-ion density remains fairly large and of the same order of magnitude as the electron density at low flowrate. 
(a) $\delta \rightarrow 1 @ 10 \mathrm{sccm}$

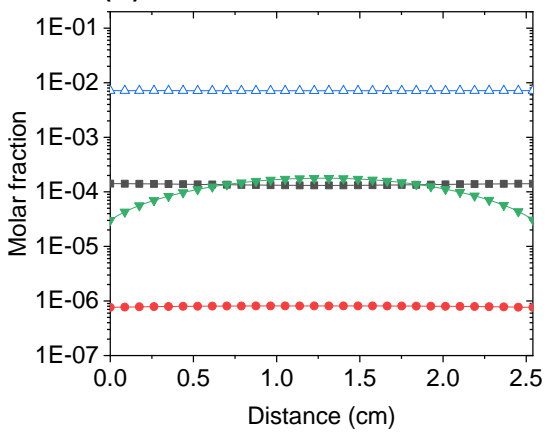

(b) $\delta \rightarrow 1 @ 100 \mathrm{sccm}$

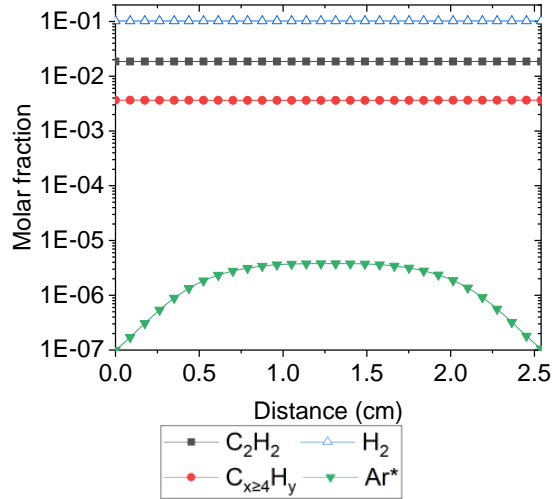

(c) $\delta \rightarrow 0$

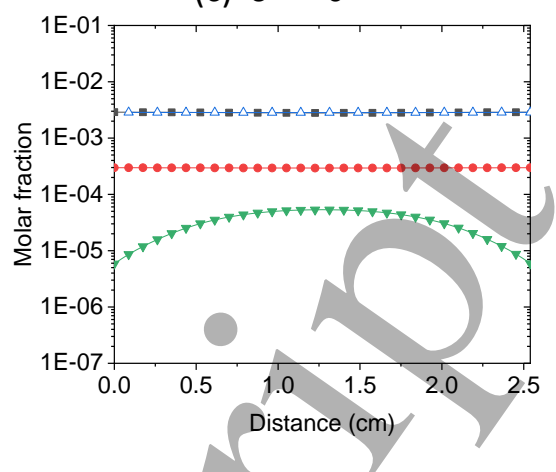

Figure 11 : molar fraction of the neutral species along the discharge different for lateral alimentation conditions

(a) $\delta \rightarrow 1 @ 10 \mathrm{sccm}$

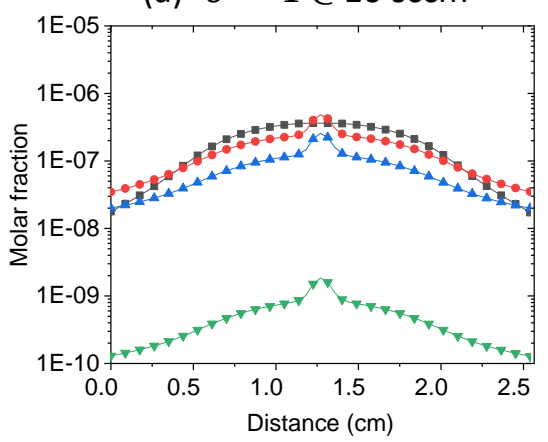

(b) $\delta \rightarrow 1 @ 100 \mathrm{sccm}$

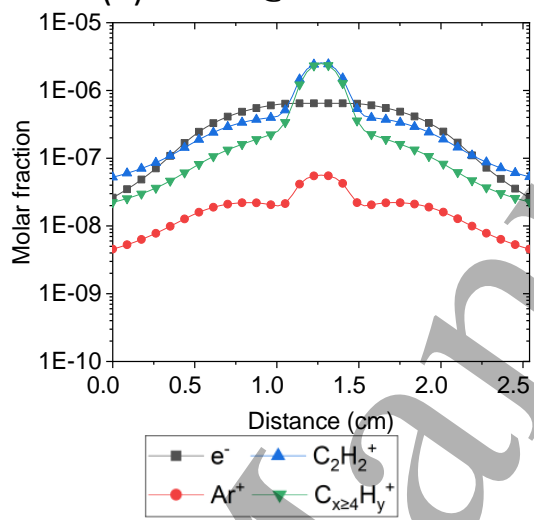

(c) $\delta \rightarrow 0$

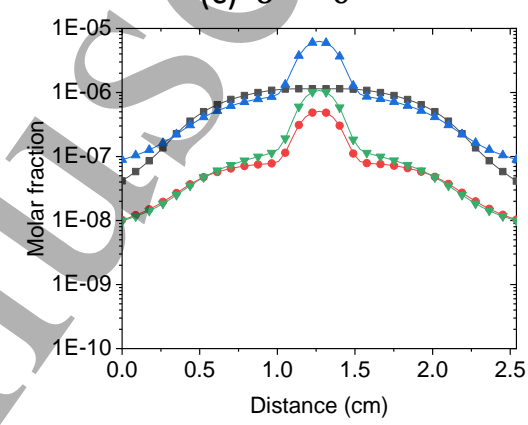

Figure 16 : molar fraction of the positives ion species along the discharge different for lateral alimentation conditions

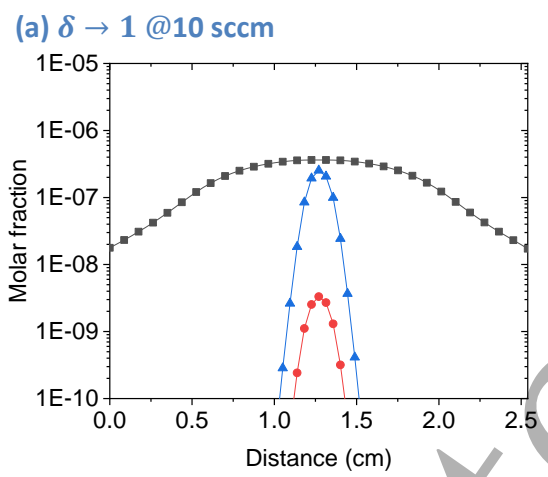

(b) $\delta \rightarrow 1 @ 100 \mathrm{sccm}$

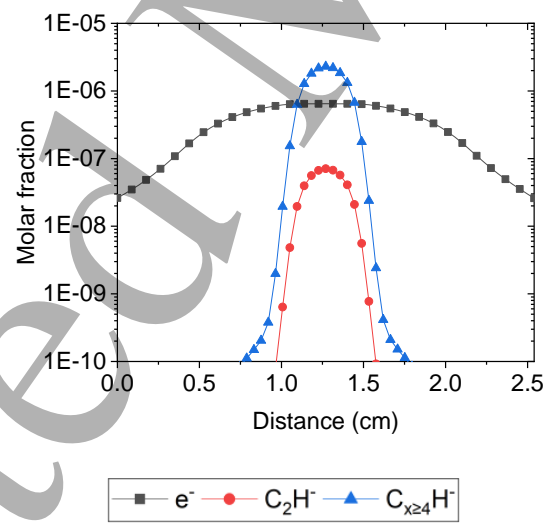

(c) $\delta \rightarrow 0$

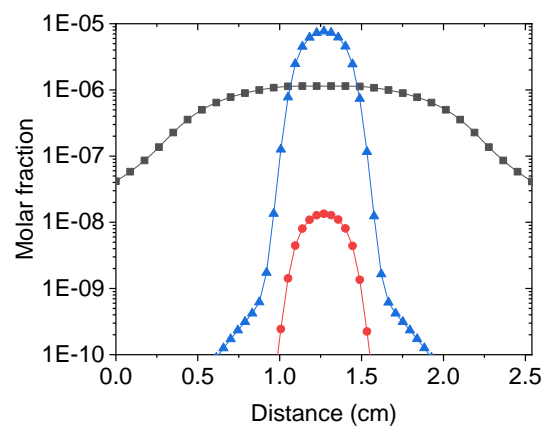

Figure 17: molar fraption of the negative ion species along the discharge different for lateral alimentation conditions

\section{Conclusion}

The modeling-based investigation of $\mathrm{Ar} / \mathrm{C}_{2} \mathrm{H}_{2}$ CCRF discharges presented in this work enables shedding light on the interplay between the discharge dynamic, the plasma kinetics and gas-flow effect in acetylene-argon plasmas. We focused on the molecular growth and nucleation stage and did not take into account solid particle formation and dusty plasma effect in this first study. The model is able to reproduce, at least qualitatively, the trends that have been observed in different experiments with respect to electron density, electron temperature, acetylene conversion yield, argon metastable 
density and large $\mathrm{HC}$ molecule concentrations. The results obtained explicitly confirm the key-role of argon metastables for all the discharge conditions and flowrate configurations investigated in this work. Whether the plasma is dominated by HC or argon ionization, argon metastables strongly contribute to the ionization and primary dissociation kinetics. This study clearly shows the major impact of the flowrate on the discharge behavior. As a matter of fact, depending on this process parameter, argon-acetylene discharges may (i) be dominated by either hydrocarbon or argon ionization kinetics, (ii) exhibit or not a strong electronegative character, (iii) show different mode of molecular growth. Basically a short residence time (large flowrate) or a large dilution conditions result in a significant concentration of acetylene in the plasma. The discharge is dominated by $\mathrm{HC}$ ionization and attachment kinetics that result in a narrow strongly electronegative plasma region at the mid gap and a significant molecular growth through neutral and ions. For large residence-time values, although the acetylene is strongly depleted in the plasma and the discharge is dominated by argon-ion, the contribution of acetylene to the overall ionization kinetics remains significant. The depletion of acetylene results however in the decrease of positive and neutral $\mathrm{HC}$ densities while negative ion concentrations remain significant. These would impact the molecular growth kinetics. The simulations performed show that showerhead and remote inlet feed gas configurations yielded similar results for $\mathrm{HC}$ species, although the discharge dynamics and hydrogen balance show significant differences between the two configurations at large flowrates. The main differences are a much larger $\mathrm{H}_{2}$ concentrations and slightly lower electron and Ar* densities in remote configuration. In fact, more than the flow configuration, an accurate description of the gas flow effect and the boundary conditions remains essential in order to achieve a satisfactory mass balance without any normalization/correction procedure that would introduce a large error on the plasma composition. From the methodological point of view, the simulation carried out in this work clearly emphasized the large stiffness that characterizes argon-acetylene plasmas and results in major difficulties in achieving the (quasi-)permanent regime. From this point of view the solution procedure developed in this study turned out to be fairly effective in treating the stiffness for all the discharge conditions considered in this work. This open up many perspectives as far as more detailed investigation of molecular growth and plasma-surface interaction over a wide range of discharge conditions is concerned. It also gives the possibility to thoroughly investigate other much longer time-scale effects such as those encountered upon particle formation.

\section{Acknowledgements}

This work was partly supported by the French National Research Agency (ANR) through the MONA project (ANR-18-CE30-0016). One of us $(\mathrm{KH})$ is grateful to Institut Universitaire de France for its support.

\section{References}

[1] A. Grill, B.S. Meyerson, V.V. Patel, Ibm Journal of Research and Development 34 (1990) 849-

857.

[2] Y. Watanabe, Plasma Physics and Controlled Fusion 39 (1997) A59.

[3] T. Uchida, K. Senda, G.K. Vinogradov, S. Morita, Thin Solid Films 281 (1996) 536-538.

[4] A. Varade, A. Krishna, K.N. Reddy, M. Chellamalai, P.V. Shashikumar, in: S. Narendranath, M.R. Ramesh, D. Chakradhar, M. Doddamani, S. Bontha (Eds.), International Conference on Advances in Manufacturing and Materials Engineering, 2014, pp. 1015-1019. 
[5] M. Weiler, S. Sattel, T. Giessen, K. Jung, H. Ehrhardt, V. Veerasamy, J. Robertson, Physical Review B 53 (1996) 1594.

[6] S. Nissen, J. Heeg, M. Warkentin, D. Behrend, M. Wienecke, Surface \& Coatings Technology 316 (2017) 180-189.

[7] A. Erdemir, I. Nilufer, O. Eryilmaz, M. Beschliesser, G. Fenske, Surface and Coatings Technology 120 (1999) 589-593.

[8] S. Vasquez-Borucki, W. Jacob, C.A. Achete, Diamond and Related Materials 9 (2000) 1971 1978.

[9] R.W. Poon, J.P. Ho, X. Liu, C. Chung, P.K. Chu, K.W. Yeung, W.W. Lu, K.M. Cheung, Nuclear Instruments and Methods in Physics Research Section B: Beam Interactions with Materials and Atoms 237 (2005) 411-416.

[10] X.-M. Liu, Q.-N. Li, X. Xu, Chinese Physics B 23 (2014) 085202.

[11] B. Kasinathan, R.M. Zawawi, Carbon-based Nanomaterials for Drugs Sensing: A Review, Trans Tech Publ, 2015, 13-39.

[12] G. Capote, G.C. Mastrapa, V.J. Trava-Airoldi, Surface \& Coatings Technology 284 (2015) 145152.

[13] E. Kovacevic, J. Berndt, T. Strunskus, L. Boufendi, Journal of Applied Physics 112 (2012).

[14] K.K. Ostrikov, U. Cvelbar, A.B. Murphy, Journal of Physics D: Applied Physics 44 (2011) 174001.

[15] G. Santoro, et al., The Astrophysical Journal 895 (2020) 97.

[16] A. Von Keudell, C. Hopf, T. Schwarz-Selinger, W. Jacob, Nuclear Fusion 39 (1999) 1451.

[17] G. Fedosenko, J. Engemann, D. Korzec, Surface \& Coatings Technology 133 (2000) 535-539.

[18] D. Ch, A. Affolter, D. Magni, H. Ch, P. Fayet, Journal of Physics D: Applied Physics 32 (1999) 1876.

[19] S. Hong, J. Berndt, J. Winter, Plasma Sources Science and Technology 12 (2003) 46-52.

[20] J. Berndt, E. Kovačević, I. Stefanović, O. Stepanović, S.H. Hong, L. Boufendi, J. Winter, Contributions to Plasma Physics 49 (2009) 107-133.

[21] S. Groth, F. Greiner, A. Piel, Plasma Sources Science and Technology 28 (2019) 115016.

[22] K. Niemi, V. Schulz-Von Der Gathen, H. Döbele, Journal of Physics D: Applied Physics 34 (2001) 2330.

[23] F. Hempel, D. Lopatik, B. Sikimic, I. Stefanovic, J. Winter, J. Röpcke, Plasma Sources Science and Technology 21 (2012) 055001.

[24] A.-P. Herrendorf, V. Sushkov, R. Hippler, Journal of Applied Physics 121 (2017) 123303.

[25] A. Baby, C.M.O. Mahony, P.D. Maguire, Plasma Sources Science and Technology 20 (2011) 015003.

[26] J. Benedikt, A. Hecimovic, D. Ellerweg, A.v. Keudell, Journal of Physics D: Applied Physics 45 (2012) 403001.

[27] A. Consoli, J. Benedikt, A.v. Keudell, Plasma Sources Science and Technology 18 (2009) 034004.

[28] F.M.J.H. van de Wetering, J. Beckers, G.M.W. Kroesen, Journal of Physics D: Applied Physics 45 (2012) 485205.

[29] S. Dap, R. Hugon, D. Lacroix, L. de Poucques, J.-L. Briancon, J. Bougdira, Physics of Plasmas 20 (2013) 033703.

[30] J. Lin, S. Orazbayev, M. Henault, T. Lecas, K. Takahashi, L. Boufendi, Journal of Applied Physics $122(2017)$

[31] V. Sushkov, A.-P. Herrendorf, R. Hippler, Journal of Physics D: Applied Physics 49 (2016) 425201.

[32] I. Stefanović, N. Sadeghi, J. Winter, B. Sikimić, Plasma Sources Science and Technology 26 (2017) 065014.

[33] D.A. Alman, D.N. Ruzic, Journal of Nuclear Materials 313-316 (2003) 182-186.

[34] J.R. Doyle, Journal of Applied Physics 82 (1997) 4763-4771.

[35] S. Stoykov, C. Eggs, U. Kortshagen, Journal of Physics D: Applied Physics 34 (2001) 2160. 
[36] D. Herrebout, A. Bogaerts, M. Yan, R. Gijbels, W. Goedheer, E. Dekempeneer, Journal of Applied Physics 90 (2001) 570-579.

[37] K. De Bleecker, A. Bogaerts, W. Goedheer, Physical Review E 73 (2006) 026405.

[38] K. De Bleecker, A. Bogaerts, W. Goedheer, New Journal of Physics 8 (2006) 178.

[39] M. Mao, J. Benedikt, A. Consoli, A. Bogaerts, Journal of Physics D: Applied Physics 41 (2008) 225201.

[40] A. Akhoundi, G. Foroutan, Physics of Plasmas 24 (2018) 053516.

[41] N. Dlimi, A. El Kebch, D. Saifaoui, A. Dezairi, M. El Mouden, Optical and Quantum Electronics 46 (2014) 47-56.

[42] I. Schweigert, A. Alexandrov, D. Ariskin, F. Peeters, I. Stefanović, E. Kovačević, J. Berndt, J. Winter, Physical Review E 78 (2008) 026410.

[43] D. Ariskin, I. Schweigert, A. Alexandrov, A. Bogaerts, F. Peeters, Journal of Applied Physics 105 (2009) 063305.

[44] I.B. Denysenko, E. von Wahl, S. Labidi, M. Mikikian, H. Kersten, T. Gibert, E. Kovačević, N.A. Azarenkov, Plasma Physics and Controlled Fusion 61 (2019) 014014.

[45] I. Denysenko, E. von Wahl, M. Mikikian, J. Berndt, S. Ivko, H. Kersten, E. Kovacevic, N. Azarenkov, Journal of Physics D: Applied Physics 53 (2020) 135203.

[46] F. Van de Wetering, W. Oosterbeek, J. Beckers, S. Nijdam, E. Kovačević, J. Berndt, Journal of Physics D: Applied Physics 49 (2016) 295206.

[47] S. Mitic, S. Coussan, C. Martin, L. Couëdel, Plasma Processes and Polymers 15 (2018) 1700152.

[48] C. Deschenaux, A. Affolter, D. Magni, C. Hollenstein, P. Fayet, Journal of Physics D: Applied Physics 32 (1999) 1876-1886.

[49] E. Gogolides, H.H. Sawin, Journal of Applied Physics 72 (1992) 3971-3987.

[50] M. Coltrin, R. J. Kee, G. H. Evans, A Mathematical Model of the Fluid Mechanics and GasPhase Chemistry in Rotating Disk Chemical Vapor Deposition Reactor, 1989.

[51] R.J.K.M.E.C.P. Glarborg, Chemically Reacting Flow: Theory and Practice, Wiley, A John Wiley \& Sons Publication, 2003.

[52] Biagi, Database, www.lxcat.net, 2021.

[53] A. Buuron, D. Otorbaev, M. Van de Sanden, D. Schram, Diamond and Related Materials 4 (1995) 1271-1276.

[54] O. May, J. Fedor, B.C. Ibănescu, M. Allan, Physical Review A 77 (2008) 040701.

[55] F. Carelli, M. Satta, T. Grassi, F. Gianturco, The Astrophysical Journal 774 (2013) 97.

[56] E. Herbst, Y. Osamura, The Astrophysical Journal 679 (2008) 1670.

[57] E.W. McDaniel, E.A. Mason, (1973).

[58] M.A. Lieberman, A.J. Lichtenberg, Principles of plasma discharges and materials processing, John Wiley \& Sons, New York, 1994.

[59] E. De Rooij, A. Kleyn, W. Goedheer, Physical Chemistry Chemical Physics 12 (2010) 1406714075.

[60] V. Garofano, R. Bérard, X. Glad, C. Joblin, K. Makasheva, L. Stafford, Plasma Processes and Polymers 16 (2019) 1900044.

[61] G.A. Bird, Molecular gas dynamics and the direct simulation of gas flows (1994).

[62] T. Novikova, B. Kalache, P. Bulkin, K. Hassouni, W. Morscheidt, P. Roca i Cabarrocas, Journal of Applied Physics 93 (2003) 3198-3206.

[63] B. Kalache, T. Novikova, A.F.i. Morral, P.R.i. Cabarrocas, W. Morscheidt, K. Hassouni, Journal of Physics D: Applied Physics 37 (2004) 1765-1773.

[64] D.P. Lymberopoulos, D.J. Economou, Journal of applied physics 73 (1993) 3668-3679.

[65] F. Greiner, J. Carstensen, N. Koehler, I. Pilch, H. Ketelsen, S. Knist, A. Piel, Plasma Sources Science \& Technology 21 (2012).

[66] V. Garofano, R. Bérard, S. Boivin, C. Joblin, K. Makasheva, L. Stafford, Plasma Sources Science and Technology 28 (2019) 055019.

[67] P. Agarwal, S.L. Girshick, Plasma Chemistry and Plasma Processing 34 (2013) 489-503. 
[68] M. Jiménez-Redondo, I. Tanarro, R.J. Peláez, L. Díaz-Pérez, V.J. Herrero, The Journal of Physical Chemistry A 123 (2019) 8135-8147.

[69] F.M.J.H.v.d. Wetering, J. Beckers, G.M.W. Kroesen, Journal of Physics D: Applied Physics 45 (2012) 485205. 